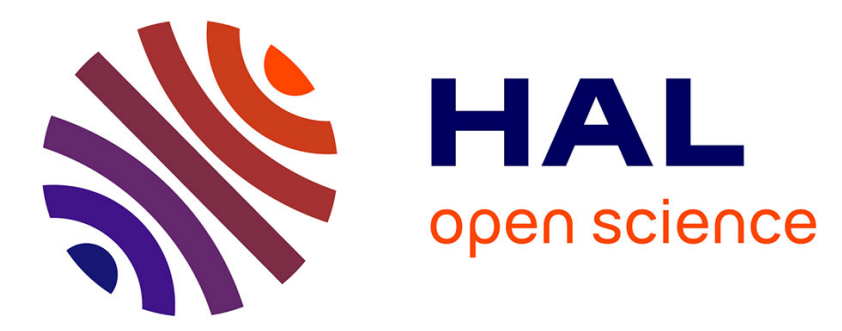

\title{
Soft matter dynamics: A versatile microgravity platform to study dynamics in soft matter
}

\author{
P. Born, M. Braibanti, L. Cristofolini, S. Cohen-Addad, D. J Durian, S. U \\ Egelhaaf, M. A Escobedo-Sánchez, R. Höhler, T. D Karapantsios, D. \\ Langevin, et al.
}

\section{To cite this version:}

P. Born, M. Braibanti, L. Cristofolini, S. Cohen-Addad, D. J Durian, et al.. Soft matter dynamics: A versatile microgravity platform to study dynamics in soft matter. Review of Scientific Instruments, 2021, 92 (12), pp.124503. 10.1063/5.0062946 . hal-03511925

\section{HAL Id: hal-03511925 \\ https://hal.sorbonne-universite.fr/hal-03511925}

Submitted on 5 Jan 2022

HAL is a multi-disciplinary open access archive for the deposit and dissemination of scientific research documents, whether they are published or not. The documents may come from teaching and research institutions in France or abroad, or from public or private research centers.
L'archive ouverte pluridisciplinaire HAL, est destinée au dépôt et à la diffusion de documents scientifiques de niveau recherche, publiés ou non, émanant des établissements d'enseignement et de recherche français ou étrangers, des laboratoires publics ou privés. 


\section{Soft matter dynamics: A versatile microgravity platform to study dynamics in soft matter}

Cite as: Rev. Sci. Instrum. 92, 124503 (2021); https://doi.org/10.1063/5.0062946

Submitted: 09 July 2021 • Accepted: 23 November 2021 • Published Online: 21 December 2021

(iD) P. Born, M. Braibanti, (iD L. Cristofolini, et al.
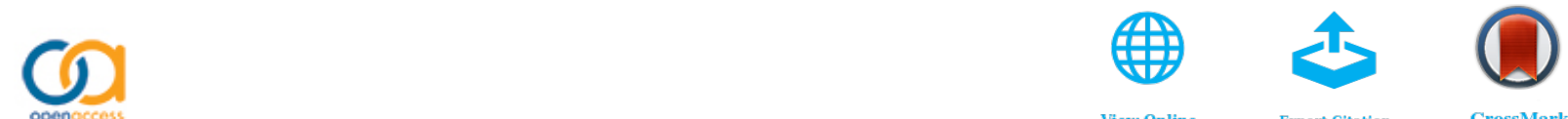

View Online

Export Citation

\section{ARTICLES YOU MAY BE INTERESTED IN}

A compact nanosecond pulse generator for $\mathrm{x}$-ray radiographies

Review of Scientific Instruments 92, 124711 (2021); https://doi.org/10.1063/5.0070794

Design\#of an insertable cone-shaped gradient coil matrix for head imaging with a volumetric finite-difference method

Review of Scientific Instruments 92, 124709 (2021); https://doi.org/10.1063/5.0060194

Novel two-tone measurement of the nonlinearity and memory effects of mixers in different amplitude regions of large carrier signals

Review of Scientific Instruments 92, 124710 (2021); https://doi.org/10.1063/5.0058668

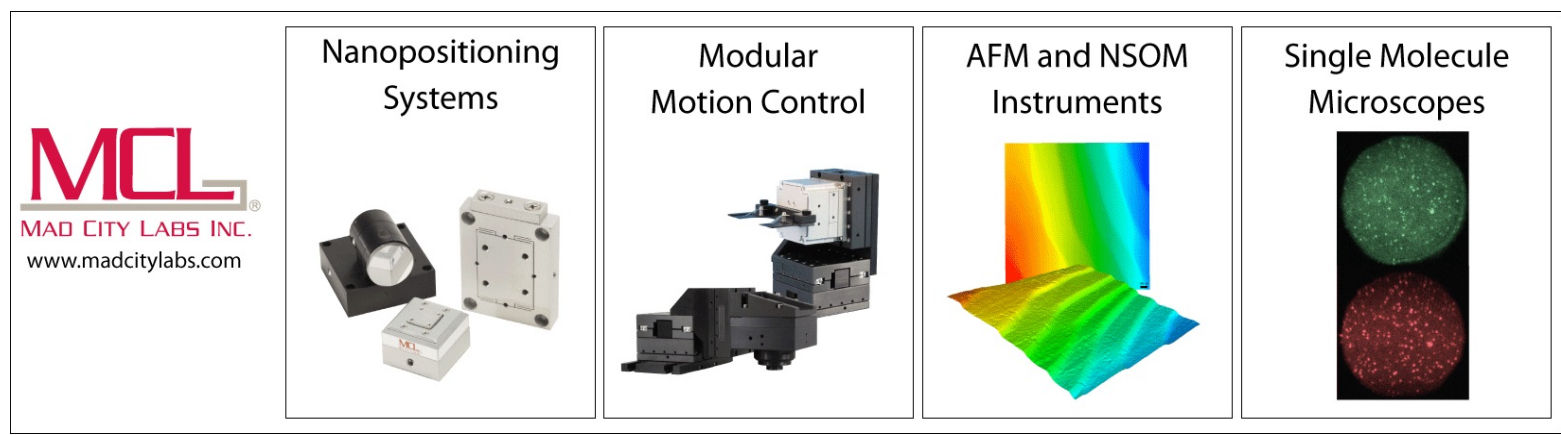




\title{
Soft matter dynamics: A versatile microgravity platform to study dynamics in soft matter
}

\author{
Cite as: Rev. Sci. Instrum. 92, 124503 (2021); doi: 10.1063/5.0062946 \\ Submitted: 9 July 2021 - Accepted: 23 November 2021 • \\ Published Online: 21 December 2021
}

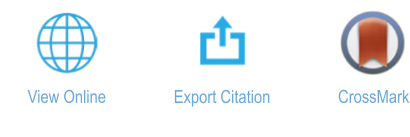
P. Born,
M. Braibanti,
${ }^{2}$ L. Cristofolini,
S. Cohen-Addad
S. U. Egelhaaf,
M. A. Escobedo-Sánchez,
R. Höhler, ${ }^{5,6}$ T. D. Karapantsios,
D. J. Durian
L. Liggieri, ${ }^{3}$
M. Pasquet, ${ }^{10}$
E. Rio, ${ }^{10}$ A. Salonen, ${ }^{10}$ M. Schröter, ${ }^{11}$ (D) M. Sperl, ${ }^{1,12}$ (D) R. Sütterlin, ${ }^{13}$ and A. B. Zuccolotto-Bernez

\begin{abstract}
AFFILIATIONS
${ }^{1}$ Institut für Materialphysik im Weltraum, Deutsches Zentrum für Luft- und Raumfahrt (DLR), 51170 Köln, Germany

${ }^{2}$ HE Space Operations BV for ESA, NL-2200AG Noordwijk, The Netherlands

${ }^{3}$ CNR - Institute of Condensed Matter Chemistry and Technologies for Energy Unit of Genoa, 16149 Genova, Italy

${ }^{4}$ Department of Mathematical Physical and Computer Sciences, Università di Parma, 43100 Parma, Italy

${ }^{5}$ Sorbonne Universités, UPMC Univ Paris 06, CNRS-UMR 7588, Institut des NanoSciences de Paris, 4 place Jussieu, 75005 Paris, France

${ }^{6}$ Université Gustave Eiffel, 5 Bd Descartes, Champs-sur-Marne, F-77454 Marne-la-Vallée Cedex 2, France

${ }^{7}$ Department of Physics and Astronomy, University of Pennsylvania, Philadelphia, Pennsylvania 19104, USA

${ }^{8}$ Condensed Matter Physics Laboratory, Heinrich Heine University, 40225 Düsseldorf, Germany

${ }^{9}$ Department of Chemical Technology, School of Chemistry, Aristotle University of Thessaloniki, 54124 Thessaloniki, Greece

${ }^{10}$ Laboratoire de Physique des Solides, CNRS UMR 8502, Université de Paris Saclay, 91405 Orsay, France

"Max Planck Institute for Dynamics and Self-Organization, 37077 Göttingen, Germany

${ }^{12}$ Institut für Theoretische Physik, Universität zu Köln, 50937 Köln, Germany

${ }^{13}$ Department TESXS Science Systems Engineering, Airbus Defence and Space, Claude Dornier Str., 88090 Immenstaad Germany
\end{abstract}

a) Author to whom correspondence should be addressed: philip.born@dlr.de

\begin{abstract}
We describe an experiment container with light scattering and imaging diagnostics for experiments on soft matter aboard the International Space Station (ISS). The suite of measurement capabilities can be used to study different materials in exchangeable sample cell units. The currently available sample cell units and future possibilities for foams, granular media, and emulsions are presented in addition to an overview of the design and the diagnostics of the experiment container. First results from measurements performed on ground and during the commissioning aboard the ISS highlight the capabilities of the experiment container to study the different materials.

(C) 2021 Author(s). All article content, except where otherwise noted, is licensed under a Creative Commons Attribution (CC BY) license (http://creativecommons.org/licenses/by/4.0/). https://doi.org/10.1063/5.0062946
\end{abstract}

\section{INTRODUCTION}

Dispersions of grains, bubbles, or droplets in a continuous phase with a different density are unstable in the presence of gravity due to drainage, sedimentation, and creaming. Density matching is not possible for foams and dry granular media but can sometimes prevent these effects for emulsions and suspensions; however, this restricts the choice of materials that can be used and can alter the physics of the system. ${ }^{1}$ Levitation methods can counterbalance gravity, for instance, using magnetic fields. ${ }^{2,3}$ However, this approach has limitations due to the requirements on field strength and due to the low temperature of the superconducting magnets in the vicinity of the sample. Performing experiments in microgravity is the method of choice to prevent drainage, sedimentation, and creaming in soft matter experiments. Such 
experiments can therefore yield valuable, well-controlled reference data.

Several platforms already exist to create microgravity conditions, each with different restrictions. Parabolic flights and drop tower experiments create microgravity only for times up to $24 \mathrm{~s}$. With sounding rockets, the experimental time can be extended up to $12 \mathrm{~min}$ depending on the rocket type. The International Space Station (ISS) provides the opportunity for microgravity experiments extending up to several months.

The experiment container presented here provides several features that, combined, distinguish it from previous setups designed to study soft matter samples in microgravity: $:^{4-12}$

1. Enables the study of slow dynamics or of statistics of rare events over months by using the ISS as the platform for microgravity.

2. Enables the study of dynamic processes inside of opaque materials, such as foams, emulsions, and granular materials, as well as inside of transparent materials by combining light scattering diagnostics with optical microscopy.

3. Enables the investigation of different samples and the use of different sample environments in a flexible manner: The processing of the samples is contained in easy-to-replace Sample Cell Units (SCUs) with up to four sample cells each, while diagnostics and data handling are provided by the experiment container.

Slow dynamics accompanied by rare dynamic events arise, for example, in glassy states of hard-sphere colloids, during foam coarsening or when gels and granular media age. Details of such processes and the scientific motivation are given in the sections on the sample cell units for the soft matter dynamics experiment (see Secs. III A and III B). Preliminary experimental results from experiments aboard the ISS are given in Secs. III A 5 and III B 5.

Optical and light scattering diagnostics are crucial for soft matter studies, ${ }^{13-17}$ even dynamics in opaque samples can be probed with light scattering. ${ }^{17-19}$ The separation of the diagnostics from the sample processing is the prerequisite for a versatile setup. The experiment container with the optical and light scattering diagnostics is described in Sec. II. The samples in self-contained SCUs can be easily replaced to allow for experiments with new materials. Future developments illustrating the versatility are sketched in Sec. IV.

The history of this Soft Matter Dynamics (SMD) experiment container dates back to the late 1990s and builds upon the "Foam Optics and Mechanics" project funded by the National Aeronautics and Space Administration (NASA) since 1992 to study coarsening and rheology for a sequence of progressively wetter foams. In 1999, a project proposal for a microgravity experiment on foam drainage and foam rheology was selected by the European Space Agency (ESA). After extensive studies, the project was reinitiated in 2007, with a new focus on foam coarsening. The scientific community in the new project named FOAM-C grew considerably, and Astrium (now Airbus D \& S) was tasked to build the experiment. In 2014, the project was again reinitiated when ESA decided to include formerly distinct projects on granular media and emulsions.

\section{THE EXPERIMENT CONTAINER}

A schematic drawing of the soft matter dynamics experiment container is given in Fig. 1. The outer dimensions of the container are $40 \times 28 \times 27 \mathrm{~cm}^{3}$. The main subsystems are the Experiment Container Controller Unit (ECCU), the sample cell carrier consisting of a rotating tray driven by a stepper motor, the sample cell units inserted to the rotating tray, and the optical diagnostics. The ECCU provides the computer and the electrical interfaces as well as the connection toward the Fluid Science Laboratory (FSL) on board the ISS.

The experiment container is mounted into a drawer that, in turn, is attached to the rest of the FSL rack through anti-vibration rubber interfaces. In addition, accelerometers are fixed to the drawer to monitor the microgravity level. The FSL rack provides the power supply and the data and video managing for the experiment container through the Video Management Unit (VMU-2). ${ }^{20}$

A two-stage system is applied to control the temperature of the experiment: the whole sample cell carrier is located inside an isothermal housing and is connected to a water cooling loop provided by the FSL. This ensures a temperature slightly lower than the target temperature for the sample cells. A variable heat source fine tunes the temperature of the sample cells in this environment; it compensates for variations of cooling water temperature and for the heat produced by the SCUs, for example, during foam generation. The temperature is measured with three sensors located between the sample cells inside each sample cell unit. The temperature is typically adjusted to $25^{\circ} \mathrm{C}$, with maximal deviations of $\pm 0.5 \mathrm{~K}$.

One of the novel features of the experiment container is the separation of diagnostics and sample processing, which ensure versatility. The experiment container itself was developed by the FOAM-C collaboration. However, the capability of the flexible sample cell carrier and the combination of light scattering and optical microscopy fostered the usage also within other communities. The details of the sample cell carrier and the diagnostics are described in Subsections II $\mathrm{A}$ and II $\mathrm{B}$.

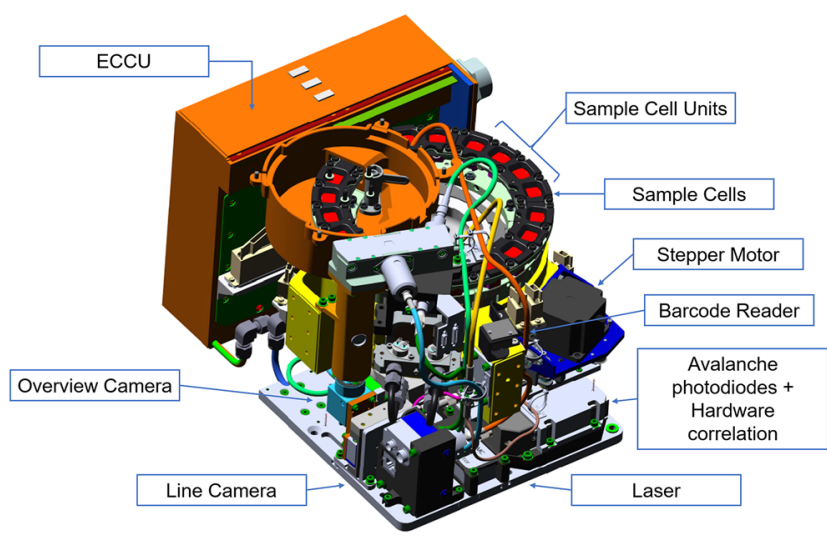

FIG. 1. Schematic representation of the interior of the soft matter dynamics experiment container. The main components are the experiment container controller unit (ECCU), the sample cells in the sample cell units, the stepper motor to drive the carousel with the sample cell units, the barcode reader to verify the sample under investigation, and the components of the diagnostics laser, overview camera, line camera, and avalanche photodiodes with a hardware-implemented intensity correlator. See text for details. 


\section{A. The sample cell carrier}

The sample cell carrier facilitates remote control of the sample in the measurement position and the on-orbit exchange of the SCUs. Up to five SCUs are mounted onto a rotating tray (see Fig. 1). Each SCU can accommodate several sample cells. The tray can be rotated such that any of the sample cells in the SCUs can be placed into the measurement position, where it can be accessed by the optical diagnostics. The rotating tray also contains a reference stop to identify the absolute position of the tray. In addition, the SCUs are equipped with Code 39 barcodes. A laser scanner (Intermec EV15) located next to the measurement position (see Fig. 1) reads the barcodes to ensure unambiguous identification of the sample under investigation. The rotating tray can be accessed through a hatch with a protective shroud in the experiment container. Manual exchange of the SCUs from the tray by the astronauts is possible through this hatch when the experiment container is withdrawn from the drawer in the Fluid Science Laboratory. The sample cell carrier has a connector at every SCU position. Communication with the SCUs via a RS422 serial bus is established through this connector so that temperature sensors can be read and each inserted sample cell can be selectively supplied with power.

\section{B. The optical diagnostics}

The SMD experiment container provides imaging and light scattering diagnostics. A schematic drawing of the diagnostic elements and how they access the sample cells is given in Fig. 2, while the actual arrangement can be inferred from Fig. 1. The light scattering diagnostics were designed to probe the inner dynamics of opaque samples using Diffusing Wave Spectroscopy (DWS ${ }^{17,18,21,22}$ ) and Time-Resolved Correlation spectroscopy $\left(\right.$ TRC $\left.^{23}\right)$ and to characterize the inner structure of the samples using Diffuse Transmission Spectroscopy (DTS, ${ }^{24}$ ). Video microscopy is used to give visual impressions and to observe structural changes with incoherent illumination. Calibration experiments and application examples of these diagnostics are given in the descriptions of the SCUs for foams and for granular media (see Sec. III).

\section{Light scattering diagnostics}

The arrangement of the components of the light scattering diagnostics is illustrated in Fig. 2. A three-faced prismatic structure is engraved into the front of the transparent sample cell for illumination with a laser, detection of backscattered light with avalanche photodiodes, and detection of backscattered light with a line camera. The faces of the prismatic surface structure are at an angle of $25^{\circ}$ with the surface normal of the sample cell. The illumination and backscattering directions are thus at an angle of $33^{\circ}$ with respect to each other. In transmission, the flat transparent cell walls allow light detection with avalanche photodiodes. Transmitted light is thus detected at an angle of $25^{\circ}$ with respect to the illumination direction.

A $532 \mathrm{~nm}$ diode-pumped laser with $200 \mathrm{~mW}$ power (Cobolt $\mathrm{AB}, \mathrm{DPL}$ 532) is used as a light source. An external attenuator is used to adjust light intensity without affecting the laser stability. A polarization-maintaining single-mode fiber and a collimator are used to illuminate a $2.1 \mathrm{~mm}$ spot on the sample surface.

Fiber optics are also used to collect the backscattered and transmitted light. A multi-mode fiber with collection optics guides the light to the high speed line camera (Basler racer, raL2048-48gm,

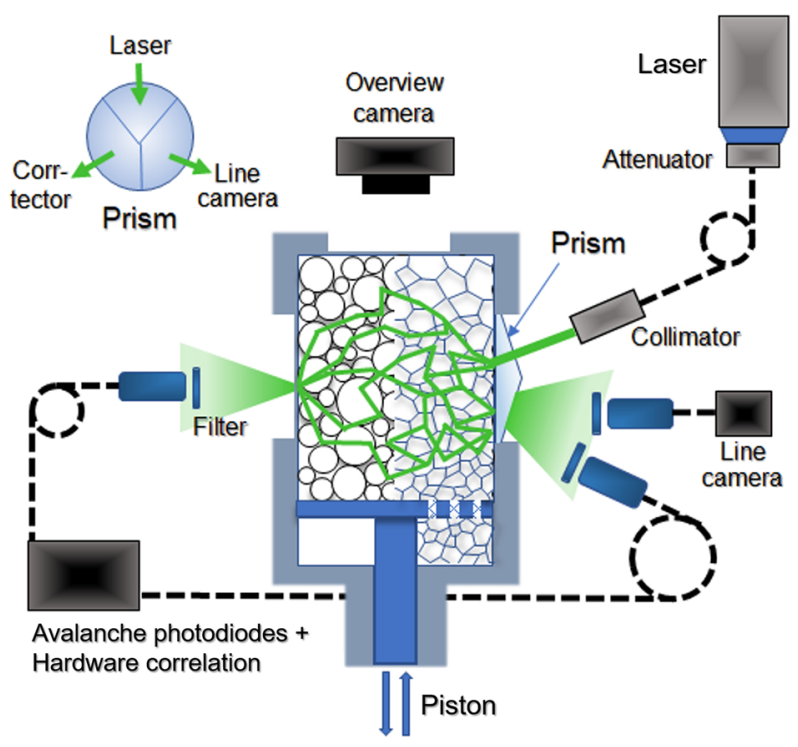

FIG. 2. Schematic representation of the optical diagnostics of the soft matter dynamics experiment container. Central to the scheme is a sample cell as used in the experiments described in Sec. III. Up to four of such sample cells form a sample cell unit located on the carousel as depicted in Fig. 1. One sample cell at a time can be made accessible to the diagnostics by moving it with the carousel to a measurement position. The intensity of the laser light is set by a motorized attenuator before being guided and collimated onto the sample cell. The sample cell body is made out of a transparent polycarbonate (PC) or a cyclic olefin copolymer $(\mathrm{COC})$ and comprises a three-faceted prismatic interface for the illuminating light and the light being backscattered from the sample. In the upper left, a front view of this interface is given. Two collimators oriented normal to their respective facets collect the light for the line camera and the avalanche photodiodes in the backscattering direction. In the transmission direction, the collimator collects light transmitted through the flat cell wall and feeds it into the photodiodes. Polarizers and bandpass filters ensure that only multiple scattered laser light is fed into the fiber optics. The overview camera with a microscope objective images the sample through the top surface of the sample cell. The sample is illuminated by three light emitting diodes (LEDs) mounted in the SMD experiment container for imaging (LEDs not shown). A piston mechanism can be used for foaming or compressing the samples, respectively.

2048 pixel, 8 bit) for multi-speckle DWS. This camera records up to 10000 lines per second.

Single-mode fibers collect the light for the avalanche photodiodes (ALV Corrtector, a hardware-implemented intensity correlator equivalent to the ALV-7004/USB with integrated avalanche photodiodes) for single-speckle diffusing wave spectroscopy (DWS). Polarizers are used to ensure that only multiply scattered laser light is collimated into the fiber, while a bandpass filter blocks the incoherent LED light used for video microscopy (see Sec. II B 2). Four avalanche photodiodes are illuminated in each scattering direction, backscattering or transmission, whose signals are fed into the input channels of the hardware-implemented intensity correlator. The hardware correlator can be remotely selected to calculate the auto- or crosscorrelation of the input intensity traces. The minimal delay for calculating the correlation is $12.5 \mathrm{~ns}$. The monitoring and the correlation channels of the hardware correlator as well as the intensity traces are read out and stored so that the time segments used in the calculation of the hardware correlation as well as arbitrary delay times 
in software-calculated correlation functions can be chosen during post-processing.

\section{Video microscopy}

An optical microscope with a camera (Pointgrey P/N FL3-GE50S5M-C, equipped with a Sony ICX655 sensor with global electronic shutter to control exposure) is used to image the sample through the top window of the sample cells. The custom-made apochromat microscope objective attached to the camera is corrected for the $4 \mathrm{~mm}$ thick top window to the sample cells and provides a focal length $26.6 \mathrm{~mm}$ with a numerical aperture of 0.07 . The microscope is mounted on a translation stage, which provides a scanning of the focal plane through the sample over $15 \mathrm{~mm}$ with a resolution of 100 steps $/ \mathrm{mm}$. The depth of field is $300 \mu \mathrm{m}$, as determined with a reference sample (see Fig. 3). The camera chip provides 2448 $\times 2048$ pixel $^{2}$, each corresponding to $5.9 \times 5.9 \mu \mathrm{m}^{2}$ in the object plane. The full field of view (FOV) of the microscope is 14.4 $\times 12.2 \mathrm{~mm}^{2}$ (see Fig. 3), which can be recorded at a rate of $8 \mathrm{fps}$. Higher frame rates are possible with smaller regions of interest. Blue light emitting diodes (LEDs) illuminate the samples through the transparent walls of the sample cells. Their intensity can be individually adjusted to create an approximately homogeneous illumination of the sample and can be synchronized with the camera exposure to minimize heat input.

The optical microscope with the overview camera is presently used to inspect uniformity of foam samples after their production and to determine the distribution and average bubble size. Since foams strongly scatter light, only bubbles close to the surface can be seen. Information about a bulk average of the bubbles size can be obtained using the diffuse light transmission probe presented in
Sec. III A 3. Moreover, the optical microscope is used to visually inspect the granular packings.

\section{THE SAMPLE CELL UNITS}

The experiment container provides only the optical diagnostics. The preparation and processing of the samples thus are provided by the sample cell units (SCUs). Some restrictions to the design of SCUs are given by the rotating tray with its power and communication interface and the interface to the optical diagnostics. The setup still provides flexibility to include very different functionalities. Presently, two SCUs to study foams and granular media are used on board the ISS. The foam SCU was developed by a scientific collaboration on the hydrodynamics and coarsening of wet foams (FOAM-C). The granular media SCU was developed by a collaboration on compaction phenomena in dense granular media (CompGran). A third SCU to study foam rheology is developed, and conceptual studies to use foam SCUs to study emulsion are ongoing within a collaboration on particle stabilized emulsions and foams (PASTA). The requirements and the scientific background of the material classes differ considerably and will be independently discussed in Secs. III A and III B.

\section{A. Foam sample cell units}

Aqueous foams are a dispersion of gas bubbles in water. They are metastable systems and evolve with time via different processes: gravity-driven drainage of the liquid between bubbles, coalescence due to the rupture of liquid films between bubbles, and coarsening due to gas transfer between bubbles driven by differences in internal pressures. ${ }^{26-28}$ Coalescence and coarsening both lead to an a)

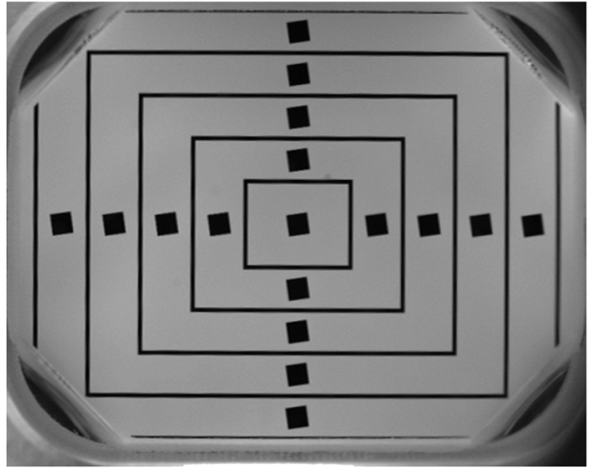

b)

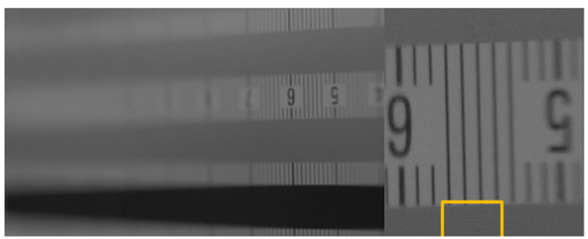

c)

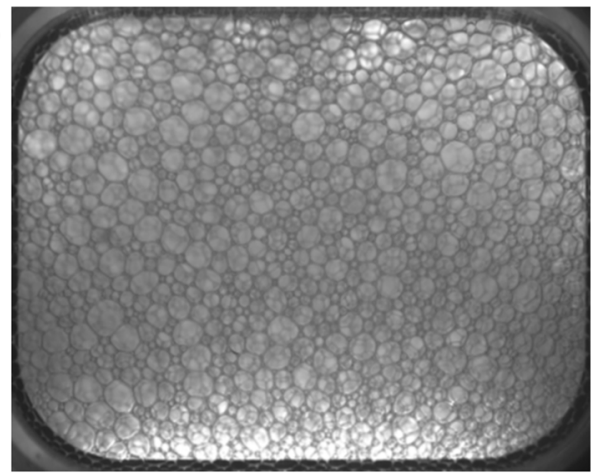

d)

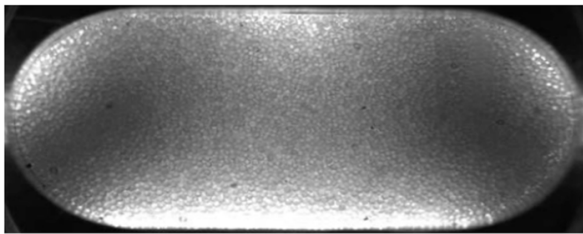

FIG. 3. Calibration images from the overview camera. (a) $14.4 \times 12.1 \mathrm{~mm}^{2}$ FOV of the microscope. The outermost (partial) rectangle corresponds to $12.5 \times 10.0 \mathrm{~mm}^{2}$ in the object plane. Pictures from on-orbit commissioning of the SMD container with a reference SCU. ${ }^{25}$ (b) Depth of field sample in the reference SCU. The yellow rectangle indicates the depth of field of $300 \mu \mathrm{m}$. The calibration of the camera on board the ISS allows accurate bubble size determination in space and calibration of the DTS data. (c) Foam image obtained after foaming in a foam SCU on ground. (d) Granular sample at 59\% volume filling in the granular media SCU aboard the ISS. Adapted with permission from Sütterlin, 69th International Astronautical Congress (IAC 2018), Bremen, Germany. Copyright 2018 International Astronautical Federation. 
increase in the average bubble radius with time and are not easy to distinguish. Drainage removes liquid from the foam and influences both coarsening and coalescence. Experiments in microgravity can thus clarify the time evolution of foams solely due to coarsening by suppressing the drainage that rapidly dries out foams on the Earth. Foams with a wide range of liquid fractions can thus be studied over long times. Coalescence can be suppressed by the choice of surfactants adsorbed to the gas-liquid interfaces, and it tends to be less frequent in wet foams compared to dry foams.

\section{Scientific motivation}

The foam project is focused on coarsening, which proceeds over long times. This is of interest because many studies have shown that coarsening of foams of arbitrary initial structure leads to a statistical self-similarity, where the bubble size distributions at different times collapse on a master plot if the sizes are scaled by their average values. ${ }^{24}$ In this so-called scaling state, the average bubble size grows with foam age following a power law whose exponent depends on the liquid fraction. Theoretical models of this process are so far available only in the limit of dry foams (von Neumann's law) and for highly dilute bubble dispersions (Lifshitz Slyozov theory). ${ }^{26-28}$ Aging of foams and the existence of a scaling state at intermediate liquid fractions is not yet understood, and this is a topical field of interest. This context explains the need of experiments over long periods of time in microgravity.

\section{Description of the foam SCU}

The sample cell units for experiments of foam coarsening contain four hermetically sealed sample cells equipped each with a magnetic piston that can be actuated magnetically to produce foam. The piston oscillates in the lower region of the cell with a height of $51.5 \mathrm{~mm}$. The upper part of the cell is used for the measurements; its dimensions are $11.25 \mathrm{~mm}$ in the beam direction and 14.25 and $14.5 \mathrm{~mm}$ in the perpendicular directions. A sketch of the sample cell is given in Fig. 4.

The sample cells are made of cyclic olefin copolymer (COC), a plastic material that exhibits reduced permeability to aqueous solutions. Figure 5 shows the permeability of COC as compared with the permeability of polycarbonate (PC), which is used for non-volatile samples such as granular materials. The diffusion rate of water out of COC cells is about 50 times smaller than the diffusion rate for PC cells, with a loss of $2.5 \mathrm{mg}$ of water in 90 days for COC cells and $100 \mathrm{mg}$ for PC cells in the same period of time as indicated in Fig. 5.

The foam sample cells were stored in sealed aluminum bags with a water vapor saturated environment to avoid potential liquid loss from the cells. This countermeasure was shown to completely prevent the loss of water by diffusion from the sample cells.

Every sample cell unit has three PT1000 temperature sensors. For the foam SCUs, these are located between the four samples cells. Additionally, one of the foam SCUs contains a humidity sensor to detect leakage from foam samples.

\section{Use of the diagnostics}

The overview camera provides images of the foams that are analyzed using an image processing algorithm that extracts the bubble size distribution. While this method is reliable for large bubbles typically found in well-aged foams, very small bubbles initially present

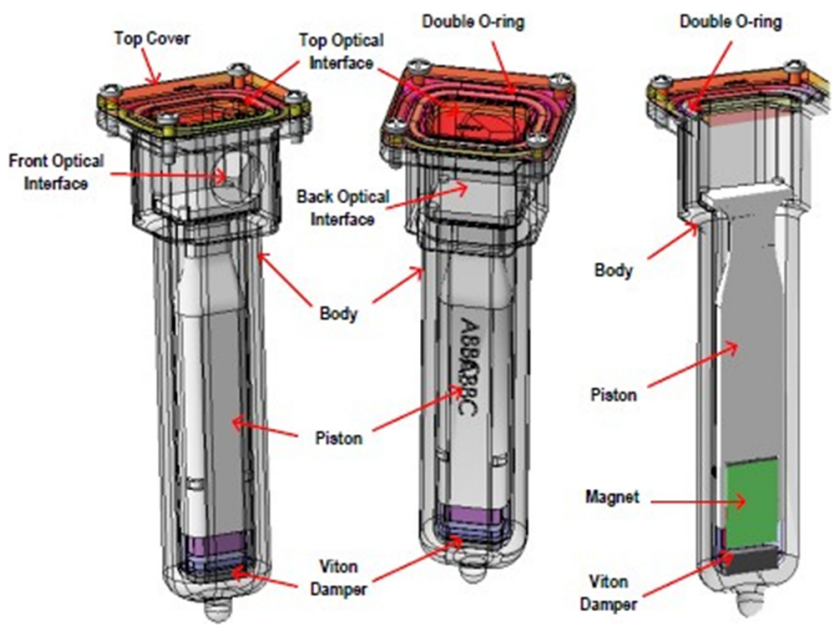

FIG. 4. Three views of a foam sample cell. The top cover and O-ring seal in a well-defined quantity of gas and liquid. Each cell also contains a mobile piston that contains a magnet (shown in green) and that can be moved periodically by an external magnetic field to create the foam. The foam in contact with the top cover can be studied by the overview camera, while laser light transmission and backscattering use the indicated "front optical interface" and "back optical interface."

after forming a fresh foam may escape detection. Reliable sizing of bubbles is possible for bubble diameters of at least ten pixels under the challenging imaging conditions of the dense bubble arrangement in the foam coarsening experiments for bubbles with more than $100 \mu \mathrm{m}$ diameter.

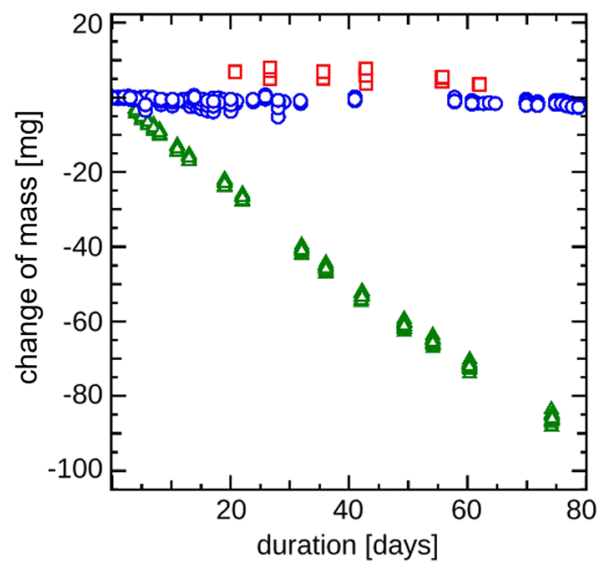

FIG. 5. Evolution of foam sample cell (SC) mass over time depending on the SC material and storage conditions. Ensembles of polycarbonate (PC) (red squares and green triangles) and cyclic olefin copolymer (COC) (blue circles). SCs were filled with water and stored at $20^{\circ} \mathrm{C}$ in wet ( $99 \%$ relative humidity, red squares) and ambient ( $55 \%$ relative humidity, green triangles and blue circles) conditions. The wet packed polycarbonate SCs gained a fixed mass due to saturation of PC with humidity, while the polycarbonate SCs under ambient conditions continuously lost mass due to permeation of water vapor from the inside of the SC to the external environment until they dried up. In the same ambient conditions, COC SCs on average lost less than $2 \mathrm{mg}$ of water ( $\sim 0.05 \%$ of SC internal volume) in 80 days. 
A complementary diagnostic called diffuse transmission spectroscopy (DTS) does not have this restriction: Measuring the average intensity of diffuse light transmitted through the sample using the photodiode gives access to the scattering mean free path $\ell^{*}$ of the photons traveling through the sample ${ }^{29}$ after calibration against imaging data. DTS is also complementary in that it measures a bulk average of the bubble size, in contrast to surface observations.

Time-resolved information about intermittent dynamics in the sample is obtained using the line camera that records multiple speckles in a nearly backscattering direction. In this geometry, most information is sampled at depths inside the sample of around three times $\ell^{*}$, close to the entrance window of the laser light. ${ }^{30}$ This is crucial for identifying individual local structural changes, such as bubble rearrangements. This configuration has been used successfully in previous ground-based investigations of dynamics in dry foams. ${ }^{31-33}$

From the line camera data, the time-resolved intensity autocorrelation function is calculated. It is a generalization of the timeaveraged correlation function commonly used in the dynamic light scattering literature, ${ }^{34}$

$$
g_{2}(\tau)=\frac{1}{\beta}\left(\frac{\langle I(t) I(t+\tau)\rangle}{\langle I(t)\rangle^{2}}-1\right) .
$$

Here, $\beta$ is a parameter depending on experimental details such as the number of speckles detected per pixel, and the brackets \langle\rangle represent an average over time in the present case. We use the fact that $g_{2}$ must converge to 1 in the limit $\tau \rightarrow 0$ to estimate $\beta$ from the experimental intensity data. This yields the following expression for $g_{2}$, which is generalized to the case of time-dependent multispeckle detection and where averages \langle\rangle$_{p}$ are performed over pixels of a given line camera image, not over time. Its implementation is explained in more detail in a previous publication,

$$
g_{2}(t, \tau)=\frac{\langle I(t) I(t+\tau)\rangle_{p}-\langle I(t)\rangle_{p}^{2}}{\left\langle I(t)^{2}\right\rangle_{p}-\langle I(t)\rangle_{p}^{2}} .
$$

To determine the quantities $\langle I\rangle_{p}$ and $\left\langle I^{2}\right\rangle_{p}$, the pixel averages are taken over speckle patterns both at times $t$ and $t+\tau$ to make them fully representative of the intensity signal in the time interval $[t, t+\tau]$. The analysis based on Eq. (2) is a variant of the time-resolved correlation (TRC) using a robust normalization scheme.

TRC is used to study the temporal correlations, rearrangement events, and the speed of bubble motion during an event. This is done by measurements of the temporal correlation $g_{2}(\tau, t)$ of the speckle pattern for a specified time lag $\tau$ vs time $t$.

In addition to TRC, foam samples are studied by conventional DWS measurements in transmission and backscattering using the photodiodes and hardware correlator. This approach only offers time-averaged information about the dynamics, but DWS can be a useful complement to TRC since the hardware correlator achieves delay times down to $12.5 \mathrm{~ns}$, while delay times are limited to $0.1 \mathrm{~ms}$ by the line camera used for TRC.

\section{Performance tests and ground-based measurements}

Optimal foaming and illumination settings were elaborated during ground-based experiments using the model SMD experiment

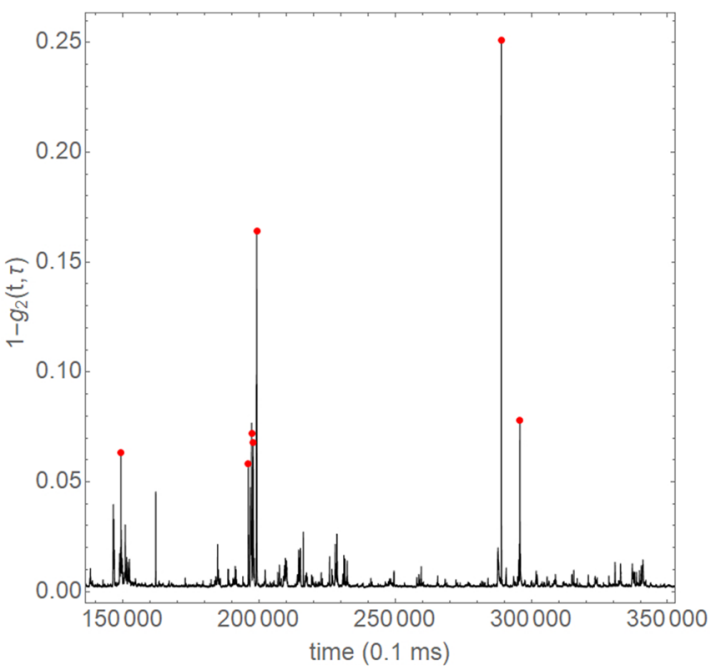

FIG. 6. The time-resolved intensity autocorrelation function $1-g_{2}(t, \tau)$ obtained with the foam SCU is plotted vs time in units of $0.1 \mathrm{~ms}$, which is the delay between successive images of the line camera. This representation emphasizes the high time resolution of the experimental setup. The signal is a measure of the average relative displacements of bubbles in the scattering volume on the time scale of the delay time. The delay time is set to $\tau=0.8 \mathrm{~ms}$. Red dots highlight prominent peaks of the intermittent dynamics. The sample liquid fraction is 0.15 and the foaming solution contains the surfactant TTAB.

container called "Elegant Bread Board" (EBB) at the Airbus Defence and Space site in Friedrichshafen, Germany.

DWS and TRC experiments showed performance comparable to those of standard laboratory DWS setups. Examples for DWS measurements are discussed in Sec. IV B (see Fig. 15). Figure 6 shows typical TRC data from a sample with a liquid fraction of 0.15 , acquired using the line camera at an acquisition rate of $10 \mathrm{kHz}$, with a delay time of $0.8 \mathrm{~ms}$. In the representation of $1-g_{2}(t, \tau)$, a signal close to zero indicates that the sample is almost static on the selected delay time scale. Intermittent peaks indicate transient rearrangement events in the bubble packing. Such dynamics have been seen in other ground experiments using TRC ${ }^{32,33}$ and Speckle visibility Spectroscopy (SVS) ${ }^{31,35}$ to probe bubble rearrangement dynamics during coarsening of dry or moderately wet foams.

\section{Preliminary microgravity results}

In the first batch of foam samples, foams made with solutions of $5 \mathrm{~g} / \mathrm{l} \mathrm{TTAB}$ (tetradecyl-trimethyl-ammonium bromide) in water, alone or with $0.2 \mathrm{~g} / \mathrm{l}$ dodecanol at different liquid fractions between 0.15 and 0.40 , were produced and bubble growth and rearrangement dynamics were studied. A typical example of a wet foam is shown in Fig. 7. On the Earth, such a foam is destabilized by drainage in a few minutes and the bubbles become polyhedral. The experiments on the ISS enabled measurement of the aging of such foams with spherical bubbles over several days.

The bubble growth due to coarsening was studied in the ISS by observations both with the overview camera and with DTS. The two techniques are complementary because the overview camera observations give the distribution of bubble sizes but only close to the 


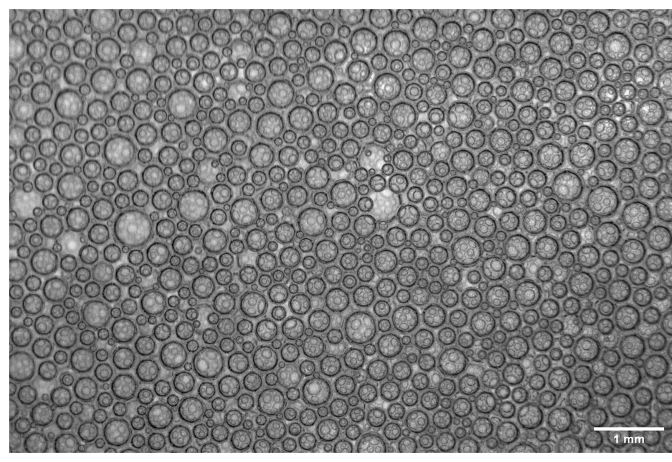

FIG. 7. Foam at a liquid volume fraction of 0.325 , observed using the overview camera $2 \mathrm{~min}$ after production. The average bubble radius is close to $100 \mu \mathrm{m}$.

surface. DTS yields information about the average bubble size in the bulk foam from the light transmission of the sample. The diffusive light propagation in bulk foam is parameterized by the photon transport mean free path $\ell^{*}$. Previous experiments have shown that this quantity is proportional to the average bubble size for bubbles larger than $\approx 100 \mu \mathrm{m}$; the dependence of the coefficient of proportionality on the liquid fraction has also been determined experimentally for liquid fractions relevant to the experiments aboard the ISS. ${ }^{29}$

The theoretical description of diffusive light transmission through an ideal slab of multiple scattering material with thickness $L$ and infinite lateral extent is well established. ${ }^{29}$ In the diffusive regime, transmission depends on the ratio $\ell^{*} / L$, with a correction term that is a function of the optical boundary conditions and the liquid fraction..$^{29}$ However, the sample cells of the foam SCUs do not have an ideal slab geometry and a fixed fraction of the incident light is lost on the sides of the cell. In addition, the light collection and illumination optics efficiencies depend on the coupling of the optical fibers.

Therefore, the DTS measurements are calibrated using the measured transmitted intensity at a known bubble diameter as determined with the overview camera. Complementary measurements with a ground-based copy of the SMD experiment container with a foam sample cell filled with calibrated latex bead suspensions are ongoing. The optical boundary conditions and the efficiencies will be determined with precisely known $\ell^{*}$-values of the samples.

The transmission diagnostics provide bubble diameters during the initial foam ages, where the bubble sizes are of the order of $100 \mu \mathrm{m}$ and difficult to detect with the overview camera up to bubble size where $L / \ell^{*}$ is of the order of 10 , where bubbles are easily detected by the overview camera. The data in Fig. 8 illustrate the excellent agreement between these two diagnostics, validating the determination of the bubble growth over more than three decades in time. The agreement of the two datasets on bubble size scales where accurate measurements are possible with both techniques, transmission measurements and camera observation, indicates that there is no significant difference between the bubble growth near the cell window and the bubble growth in the bulk. Note also the long duration of these measurements, which would not be possible on a sounding rocket.

The rearrangements detected in the foams appear qualitatively different from those occurring in the very dry foams on

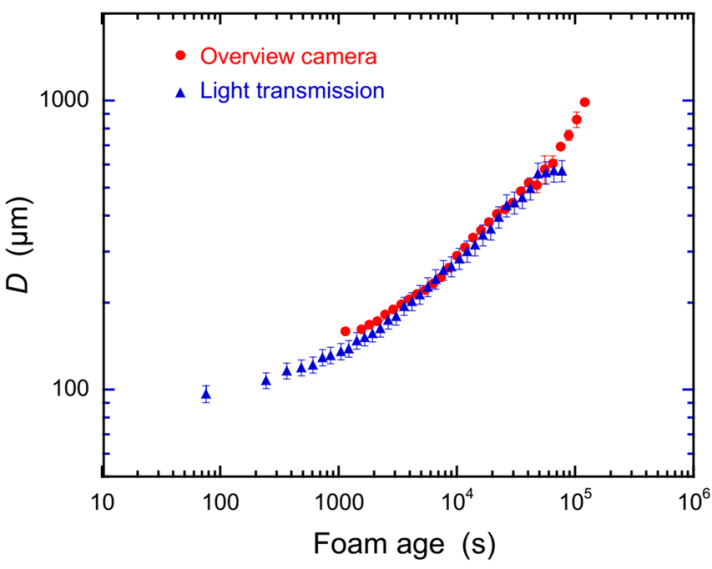

FIG. 8. The growth of the average bubble diameter in a wet foam observed using the overview camera is plotted vs foam age. The bubble diameter deduced from the light transmission signal is superposed to these data. The transmission measurement yields the average bubble size, multiplied by a scale factor that needs to be determined experimentally. This calibration is deduced from the overview camera data at a foam age of $10^{4} \mathrm{~s}$. The sample liquid fraction is 0.325 as in Fig. 7.

the Earth. These differences are presently the focus of scientific investigations.

\section{B. Granular media sample cell units}

Ensembles of athermal dry solid particles are granular media. Such media are ubiquitous in nature as well on the Earth as on other planets and in the interplanetary space. The physics of granular media in low gravity situations is fundamental to the formation of planets, the morphology of small interplanetary objects, and the mechanical properties of regolith on moons and minor planets. However, studying the physics of such granular media is limited on ground, as gravity causes quick sedimentation of granular particles and the formation of pressure gradients.

\section{Scientific motivation}

Upon tapping, ${ }^{36-38}$ thermal cycling, ${ }^{39,40}$ or just waiting, ${ }^{41}$ a granular medium will exhibit time-dependent behavior due to increasing density and increasing mechanical strength. ${ }^{42}$ The rate and extent of this consolidation depends on external factors such as tapping strength, frequency spectrum, or time. However, on the Earth, the underlying mechanism for time-dependence is the minimization of the gravitational potential of the granular particles. ${ }^{38}$ This mechanism may become negligible in situations like on asteroids and comets or within protoplanetary dust, i.e., in low gravity situations. ${ }^{43}$ In such low gravity situations, the interparticle interactions may match or even exceed the gravitational potential. Timedependent behavior of granular media in such situations may rather resemble the situation of aging colloidal gels, where the interparticle forces cause slow evolution of the particle network. ${ }^{44}$ Presently, such time-dependent behavior of granular media in low gravity is not quantified. Models to predict consolidation of media like regolith on asteroids or planetesimals have to rely on situations realizable and properties measurable on ground ${ }^{45,46}$ or on measurements on granular media prepared in microgravity with very limited waiting 
times. ${ }^{4-49}$ The granular media sample cell unit for the SMD experiment allows one to study time-dependent mechanical properties of granular media with long measurement or waiting times in microgravity. The packing density of the samples can be recorded over months, while the response of the samples to small-scale rearrangements can be repeatedly probed using the light scattering diagnostics of the SMD container.

\section{Description of the granular media SCU}

The sample cell units for experiments with granular media contain a single sample cell machined out of polycarbonate and the electronics to control the integrated actuators. The sample cell dimensions are $5 \mathrm{~mm}$ (in beam direction) times $10 \mathrm{~mm}$ (lateral direction) times a height depending on the piston position between 9 and $16.5 \mathrm{~mm}$, i.e., volumes between 0.45 and $0.83 \mathrm{ml}$. A sketch of the sample cell is given in Fig. 9. The access to the optical diagnostics is the same as for the foam sample cells. The smaller dimension of the granular media SCU in comparison with the foam SCU is apparent in the overview camera image (Fig. 3).

The piston compresses the sample with adjustable pressures between $100 \mathrm{kPa}$ and $1 \mathrm{MPa}$. In the first experiments, typically $500 \mathrm{kPa}$ were used to probe the unidirectional compressibility of the sample. The motion of the piston is monitored by a magnetic encoder (RLS RLC2IC, Renishaw) with $1 \mu \mathrm{m}$ precision to calculate the volume of the compressed sample.

Four piezocrystals are fixed inside the granular medium in the sample cell, outside of the central volume illuminated by the laser (see Fig. 9). These piezos can be set to vibrate with frequencies between 0 and $2000 \mathrm{~Hz}$. The vibration amplitudes decrease from $2.2 \mu \mathrm{m}(0-300 \mathrm{~Hz})$ to $0 \mu \mathrm{m}$ at $2000 \mathrm{~Hz}$. The piezostrokes, on the one hand, mobilize particles in dense packings ${ }^{50}$ and minimize the effects of wall friction during consolidation. ${ }^{51}$ On the other hand, the piezocrystals can be used to probe mechanical properties of the

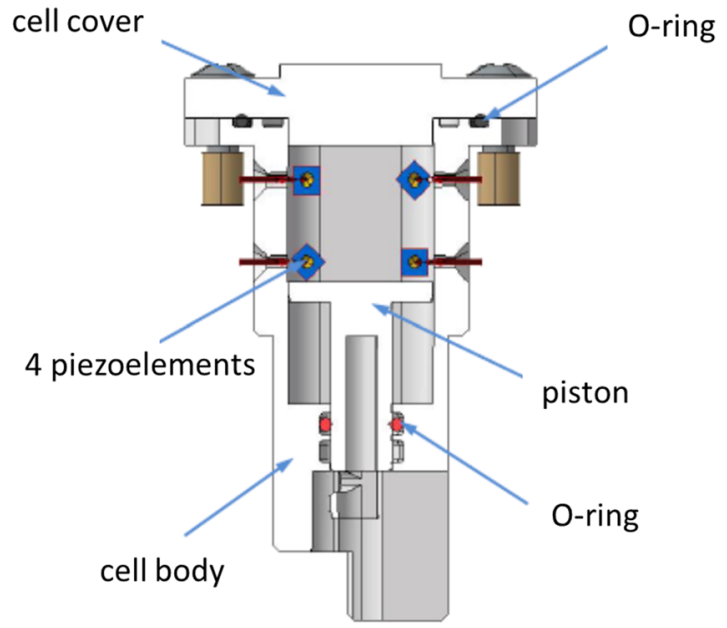

FIG. 9. Cross section of the granular media sample cell. The granular medium is located between the piston and the cell cover. The piston is motorized by a spindle drive, and a stepper motor can be used to tap the sample and to probe the packing fraction at selected pressures. Four piezocrystals inside of the granular medium create small-scale rearrangements in the granular medium and are used to probe the mechanical properties together with the light scattering diagnostics. granular medium in the sample cell together with the light scattering diagnostics. The light scattering diagnostics probe particle rearrangements on microscopic scales averaged over many particles; thus, ensemble- and time-averaged information on the response of the granular medium to the impact by the piezostrokes can be gained.

\section{Use of the diagnostics}

Light scattering probes displacements on microscopic scales. This can be used to infer information on the dynamic properties of a sample and link it to the periodic deformation. ${ }^{54-56}$ In the granular media SCU, the sample is periodically deformed by the small-amplitude vibrations of the embedded piezocrystals.

The light detected by the avalanche photodiodes of the SMD experiment in the transmission direction has propagated through the sample by multiple scattering events and thus carries information on a large volume of the sample. The time-averaged intensity autocorrelation function

$$
G(\tau)=\frac{\langle I(t) I(t+\tau)\rangle}{\langle I(t)\rangle^{2}}
$$

is calculated from the transmitted light intensity $I(t)$ by the hardware correlator integrated in the Corrtector unit with a logarithmic spacing of the delay times $\tau$. The sample changes its configuration in response to the minute impacts by the piezos, which changes the light signal transmitted through the sample. The piezos are set to expand and contract with a fixed period T. If the sample inside the sample cell would be a perfect elastic body, it would fully recover its configuration after one period of the piezos. ${ }^{54}$ If the sample irreversibly acquires configurational changes during the stroke of the piezos, it will not fully recover its original configuration during one period, and the light signal will be altered. This is reflected by the value of $G(\tau)$ at $\tau=$ T. For a perfect elastic body, $G(\mathrm{~T})$ will equal $G(0)$, as the sample reproduces exactly the same light signal after one period. We introduce a recovery level $R$ to quantify this behavior,

$$
R(\mathrm{~T})=\frac{G(\mathrm{~T})}{G(0)}
$$

A value of $R=1$ indicates a perfect recovery, while a value of $R=0$ indicates that all correlation in the light signal is lost within $\mathrm{T}$.

\section{Performance tests and ground-based measurements}

The functionality of the granular media SCU was tested using the engineering model of the SMD experiment container at B.USOC in Brussels, Belgium. Polystyrene powder (Microbeads AS, Norway) with a mean particle diameter of $140 \mu \mathrm{m}$ and a standard deviation of $17 \mu \mathrm{m}$ was used as the sample material.

Average transmitted intensities of $20 \mathrm{kHz}$ were obtained, and the intensity autocorrelation functions $G(\tau)$ are determined based on measurements of duration $10 \mathrm{~min}$. The period $\mathrm{T}$ of the piezostrokes was set to $3.3 \mathrm{~ms}$, i.e., 300 strokes/s were performed. $G(\tau)$ curves obtained under such conditions for various positions of the piston are displayed in Fig. 10(a). The recovery of correlation at $\tau=\mathrm{T}$ is clearly visible, as well as the loss of correlation during the piezostroke. 


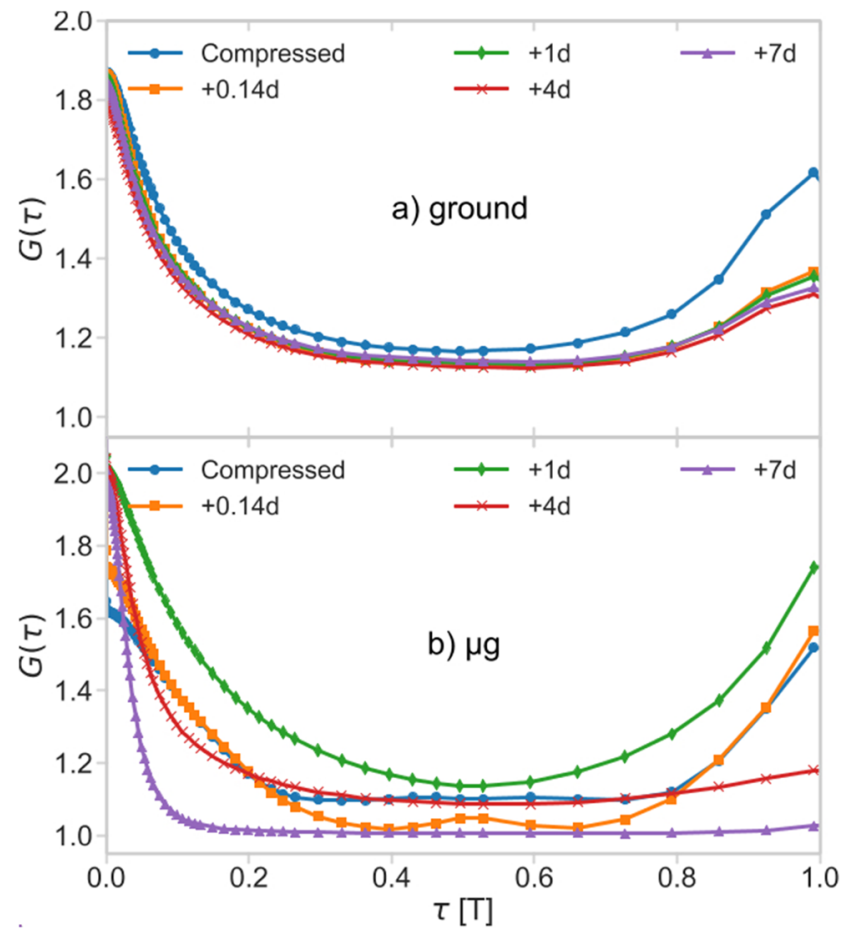

FIG. 10. Intensity autocorrelation functions $G(\tau)$ [Eq. (3)] obtained with polystyrene powder at piezostroke frequencies of $1 / T=300 \mathrm{~Hz}$ with the granular media SCU. $\tau$ is displayed in units of T. The different datasets represent measurements made at various piston positions, which are given as the distance $\Delta \mathrm{h}$ from the piston positions $\mathrm{h}$ when the sample is compressed with a pressure of $500 \mathrm{kPa}$, in units of the particle diameter $d$. The positions and the color coding in (a) and (b) are the same. (a) Ground-based results. (b) Results from measurements in microgravity on board the ISS

On ground, the dynamical properties of the sample are independent of the piston position as long as the sample cell volume is large enough. This is already apparent from the $G(\tau)$ data but can be seen even clearer from the $R$ values (Fig. 11). If the volume of the cell set by the piston is large enough, the recovery level of the sample is constant. This behavior reflects that the granular medium stays settled in a dense state confined by gravity as long as the piston leaves enough space. If the volume is further reduced by the piston, the recovery level of the sample increases.

\section{Preliminary microgravity results}

In Fig. 10(b), measurement results obtained using the same parameters as in Fig. 10(a) are shown but from measurements performed in microgravity on board the ISS. While on ground only two different shapes of the intensity correlation functions $G(\tau)$ can be observed, $G(\tau)$ does exhibit a variety of shapes with retraction of the piston. This behavior indicates that the sample is in contact with the piston at each piston position and continuously changes its elastic properties with increasing sample cell volume, in contrast to the measurements on ground.

From the respective first and last data point of each correlation function in Fig. 10, the recoveries $R$ can be calculated [Eq. (4)]. The

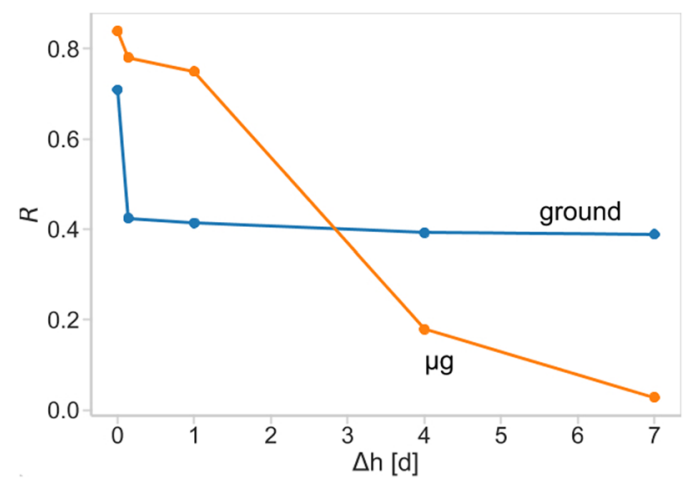

FIG. 11. Mechanical properties of the same granular medium on ground and in microgravity $(\mu \mathrm{g})$, as obtained from the measurements in Fig. 10. The individual recovery levels $R$ are the ratios of the first and last data point of an autocorrelation curve in Fig. 10 [see Eq. (4)] and are given as a function of the piston displacement $\Delta \mathrm{h}$. An $R$ value of 1 indicates that the granular medium in the sample cell fully recovers after a small perturbation of a piezostroke and thus behaves elastically, while a value of 0 indicates that all correlation is lost during a single piezostroke. The wider range of recovery levels covered in microgravity than on ground is apparent. Lines provide a guide to the eye.

$R$ values obtained in microgravity span a larger range than the $R$ levels obtained with the same parameters on ground (see Fig. 11) and continuously decrease with increasing volume of the sample cell.

This qualitative difference in the dynamical behavior of a granular sample on ground and in microgravity hints at differences in the mechanical properties in the respective situation. The timedependence of these dynamic properties probed over months for a variety of granular materials is currently in the focus of scientific investigation.

\section{FUTURE DEVELOPMENTS}

A key aspect of the soft matter dynamics experiment container is its versatility and the possibility of quick turnover of SCUs. In the following, we describe two developments for the SMD experiment that highlight this versatility. The first example, an SCU for experiments on foam rheology, is close to commissioning. The second example is a foam sample cell unit approved to study emulsions aboard the ISS.

\section{A. Foam rheology sample cell units}

The problematic situation with studying foam properties on ground is sketched already in Sec. III A. There, the problem of studying the time evolution of foams was in the focus of the experiment design. Here, studying the flow behavior of foams (rheology) is the motivation for the SCU design.

\section{Scientific motivation}

The flow behavior of foams strongly depends on the liquid volume fraction. Up to a liquid fraction $\phi_{c}$ close to 0.36 , the number of mechanical contacts among the bubbles is sufficient so that the foam can respond elastically to a small static applied stress. As long as this stress is smaller than a yield stress, the foam structure relaxes back to a reference state if the stress is released and it behaves like a soft 
solid. For applied stresses beyond the yield stress and for liquid fractions larger than the critical value $\phi_{c}$, the bubble assembly flows like a liquid.

This unjamming transition between the solid-like and liquidlike response is a topical field of research raising many questions. Many simulation studies have suggested that the length and times scales of the mechanical relaxation response diverge at the transition. ${ }^{57,58}$ However, these simulations are based on simplified 2D models of bubble interactions, which have been shown to be a very rough approximation. ${ }^{59,60}$ Therefore, experimental investigations of real foams near the jamming transition are of great interest, requiring microgravity to avoid destabilization of the samples by drainage.

Similar jamming transitions are encountered in other assemblies of randomly packed objects, from sand, clays, and emulsions to pedestrian and car traffic, and currently attract much interest. ${ }^{57,61}$ The jamming transition has similarities (and differences) with the glass transition, which is one of the open important problems in physics.

On the application side, liquid foams have a very large number of applications as detergents, as food products, in medicine, in oil recovery, in flotation of minerals, and in firefighting. These foams are wet during their creation, and knowledge of their properties will greatly improve the design of foaming processes.

Cyclic flow of jammed or unjammed foams and emulsions has been shown to induce a reorganization of their structure. Remarkably, depending on the liquid fraction and strain amplitude, this flow induced reconstruction can lead to structures that no longer change from one cycle to the next and that are able to respond elastically. This has been called an "absorbing state," 62 and this kind of flow induced self-organization offers a perspective for making new foam structures with enhanced mechanical properties. Absorbing state behavior in response to cyclic strain can be detected by a technique called diffusing wave spectroscopy $\operatorname{echos}^{63}$ and whose implementation in the SMD device has been successfully demonstrated, as presented in Sec. III B.

\section{Description of the foam rheology SCU}

The sample cell units for experiments of foam rheology contain a single sample cell equipped with two pistons, one to generate the foam as in the cells of Fig. 4 and a second piston to perform the rheology tests that squeezes part of the sample against the cell wall (see Fig. 12). This can be used to apply a controlled uniaxial strain vs time.

\section{Performance tests and ground-based measurements}

The functionality of the SCUs for foam coarsening was tested using the breadboard model at Airbus, Friedrichshafen, and, prior to the flight, using the engineering model of the SMD experiment container at B.USOC in Brussels, Belgium. Performance and validation tests of the sample cells modified for rheological experiments were carried out at the Institut des NanoSciences de Paris, which is a part of Sorbonne Université in Paris. These tests were restricted to dry foams as it is impossible to stop drainage on ground.

Figures 13 and 14 show examples of rheological experiments using the foam rheology SCU, combined with the TRC diagnostic presented in Sec. IV A 3. In the case where the applied strain

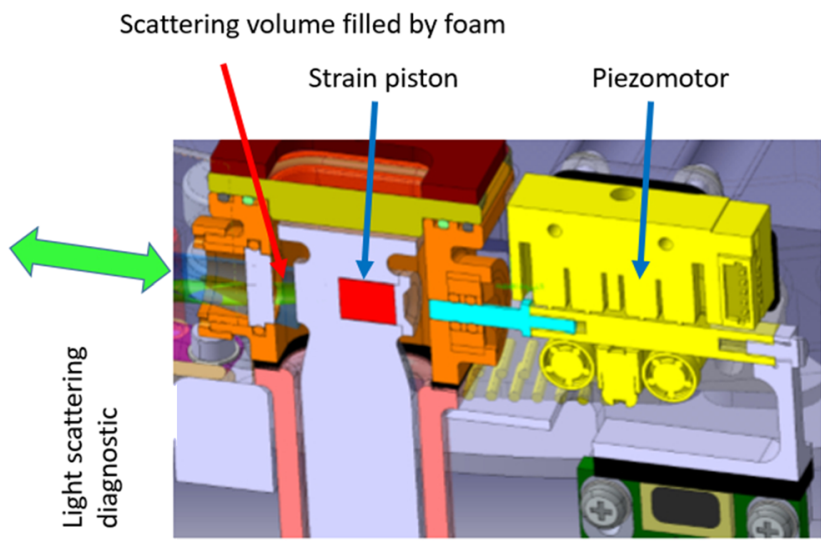

FIG. 12. A modified foam sample cell enables rheological experiments: the pictures show the top of this cell inserted into a dedicated sample cell holder unit. The shape of the cell is close to those illustrated in Fig. 4 used to study foam coarsening; the laser illumination and backscattering diagnostics are also the same for both types of cells. However, in the rheology cell, an additional piston is present to strain the sample, connected to piezomotor outside the cell via a flexible polymer membrane. Two magnets, located in the strain piston and at the end of the piezomotor, link the two mechanically.

remains below the yield strain of the foam illustrated on Fig. 13, the foam's initial microstructure is recovered when the strain is released. This is seen and analyzed using the backscattered speckle pattern, shown in the middle of the figure: it is strongly perturbed during the strain application but fully recovered when the strain is released. A similar experiment with a larger strain amplitude induces an irreversible change of the speckle pattern that reveals irreversible changes of the microstructure. This is the signature of a plastic response. Recording speckle patterns obtained in such experiments with a series of increasing strain amplitudes can thus be used to determine the yield strain of the foam. The progressive relaxation of the speckle pattern visible on Fig. 13 illustrates that viscoelastic response can also be studied.

\section{B. Emulsion sample cell units}

Emulsions are dispersions of oil drops in water. Emulsions are metastable systems because their surface energy is much larger than that of the separated phases. Like foams, they therefore evolve with time toward phase separation via gravity-driven drainage of liquid between drops, coalescence, which is the rupture of liquid films between bubbles, and coarsening (or Ostwald ripening), which is the liquid transfer between drops due to different internal pressures. In order to slow down these processes, surface active agents are used, such as surfactant molecules, polymers, proteins, or particles. A central topic in research on emulsions is the interplay of coarsening and interfacial rheology. Emulsion stability, particularly in the regime of low surfactant content, has large implications in the formulation, production, and disposal of many products of everyday use, such as food and pharmaceutics.

\section{Scientific motivation}

Stable emulsions show constant time behavior of the drop size distribution (DSD) and a uniform distribution of droplets within 

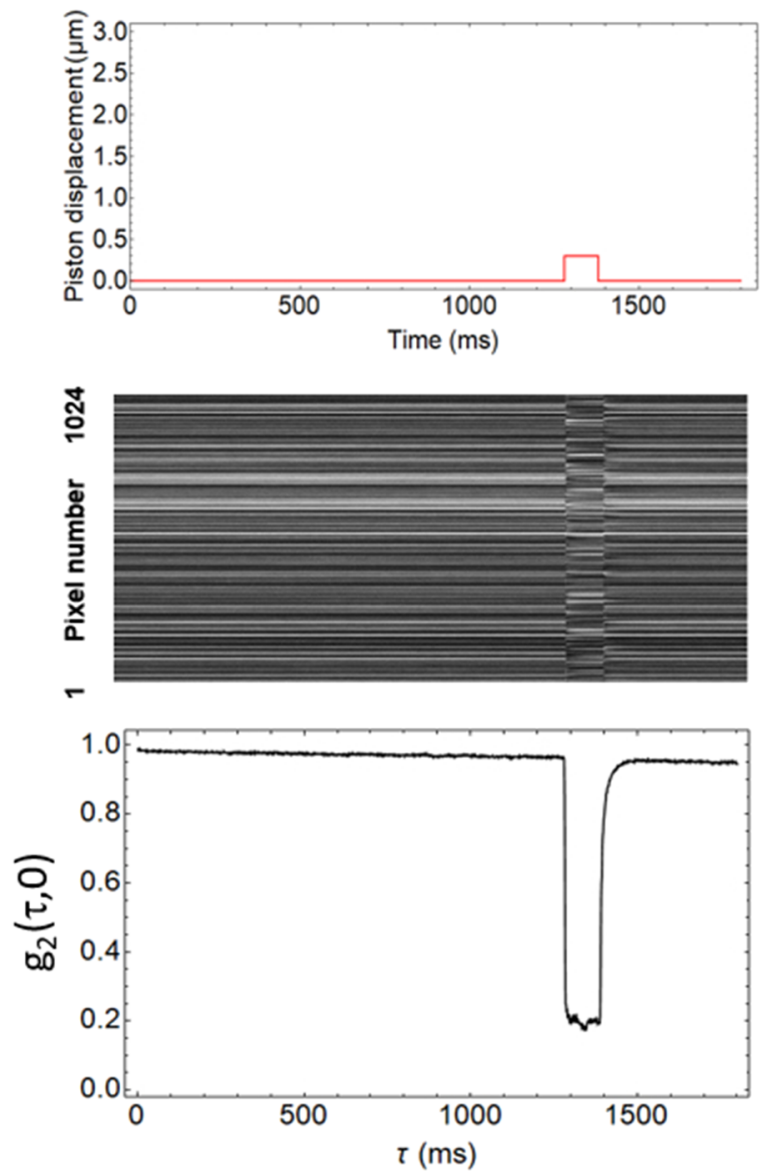

FIG. 13. These three plots illustrate an experiment using the sample cell shown in Fig. 12, allowing rheological experiments to be performed. The top plot shows the imposed piston displacement vs time. The plot in the middle shows the intensities of the backscattered light speckles recorded by the line camera as a function of time on the same time scale as the top plot. The bottom plot shows the intensity correlation between the initial speckle pattern and the one at later times, again on the same scale.

the continuous medium. Emulsions presenting a DSD in the submicrometric range are stable over a long time. In contrast, gravitydriven phase separation due to sedimentation or creaming is typical in emulsions with micrometric droplet sizes and different densities of the constituting liquids. Emulsion destabilization is directly linked to different mechanisms promoting the increase in droplet sizes: (1) coalescence, caused by the thinning of the liquid film separating droplets, a mechanism ruled by the chemico-physical properties of the surfactant layer at water-oil interfaces; ${ }^{64-69}$ (2) droplet aggregation, resulting in segregation by gravity; (3) Ostwald ripening, a non-equilibrium dynamic process that leads to the growth of larger droplets at the expenses of the smaller ones, which is ruled by the difference in Laplace pressure among droplets and thus by interfacial tension, ${ }^{70}$ by the Gibbs elasticity of the interfacial surfactant film, ${ }^{66,71,72}$ by mutual solubility between the liquids, and by the packing fraction of droplets.
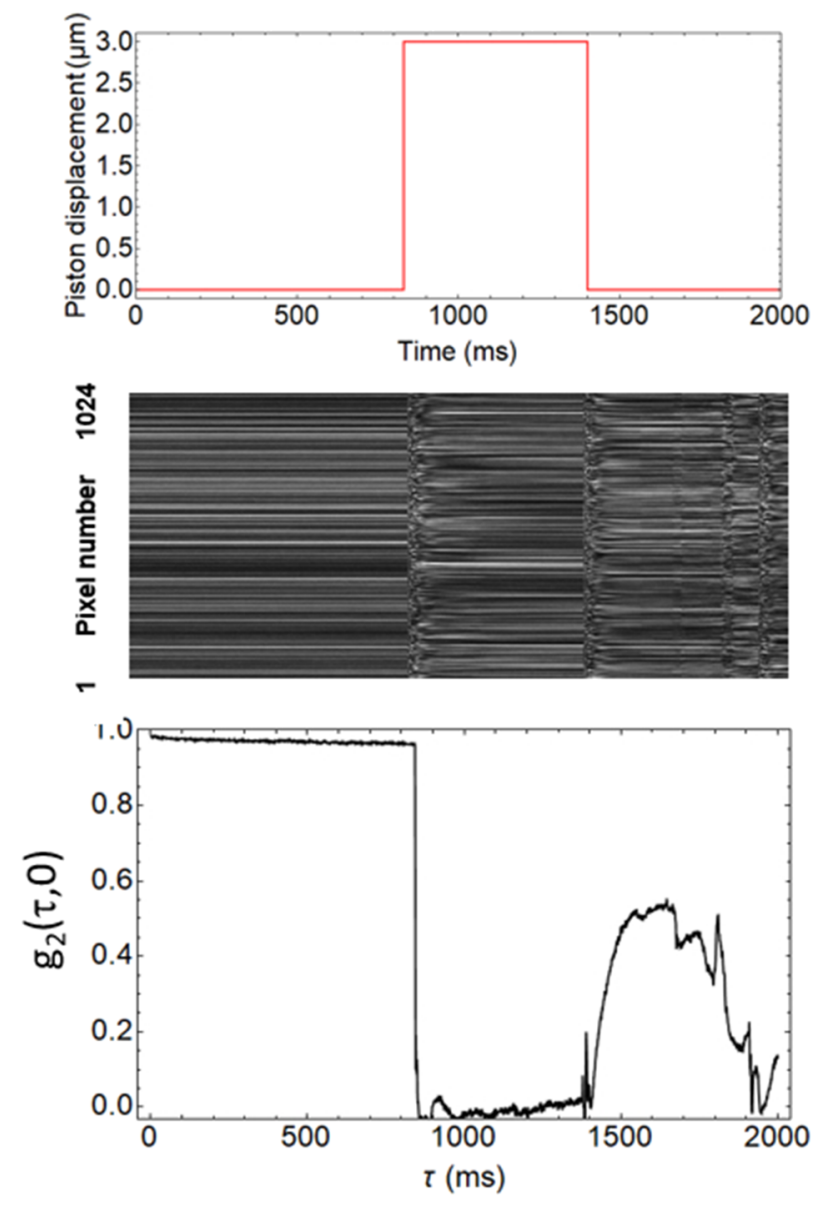

FIG. 14. These three plots illustrate an experiment using the sample cell shown in Fig. 12, allowing rheological experiments to be performed. The presentation is the same as in Fig. 13, but the amplitude and duration of the applied piston displacement are larger, leading to an irreversible mechanical response, as discussed in the text.

All these destabilization mechanisms are strongly coupled with creaming and sedimentation processes, hindering the possibility to investigate them independently on ground. Thus, due to the suppression of sedimentation or buoyancy, microgravity provides an ideal environment for an effective study of all the basic processes of coalescence, aggregation, and Ostwald Ripening and to understand their relation with the chemico-physical features of involved surfactant layers and liquids and how they influence the droplet dynamics and the onset of specific dynamical regimes, discriminating between Brownian diffusion and dynamics ruled by capillary phenomena and Marangoni forces.

\section{Description of the emulsion $S C U$}

The same SCUs as described in Sec. III A used for foams experiments will be used for experiments with emulsions. The magnetic piston designed for foam generation instead is used to emulsify oilin-water mixtures. A small gas pocket has to remain within the sample cell to compensate for volume changes of the liquids due to 
temperature changes during storage and transport of the SCUs from the manufacturer to the experiment on board the ISS. One of the main goals of the performance tests and ground-based measurements is to ensure negligible influence of this gas pocket on the experiments. In order to completely prevent the detrimental influence, Airbus has devised a special cell able to bear the related mechanical stresses without a gas pocket, but this new cell has not yet been implemented.

\section{Performance tests and ground-based measurements}

The functionality of the emulsion SCU was tested using the breadboard model at the Airbus Defence and Space site in Friedrichshafen, Germany, as well as in the laboratory at Aristotle University, Tessaloniki, Greece.

Emulsification tests aimed at determining the effects of piston frequency and emulsification time on the DSD. The piston can oscillate at frequencies between $17 \mathrm{mHz}$ and $20 \mathrm{~Hz}$. Various emulsification sequences with different oscillation frequencies and durations were tested. After each emulsification experiment, the DSD was evaluated by analyzing overview camera images of drops close to the top cell window.

The DWS performances of the SMD apparatus were evaluated on ground by analyzing the aging behavior of an emulsion right after its emulsification. The aging is characterized using the intensity autocorrelation functions $G(\tau)$ - 1 obtained from the hardware correlator in the backscattering and transmission direction at different times after the end of the emulsification process.
The sample investigated is an emulsion of medium chain triglyceride oil drops in water, stabilized by a food grade surfactant in concentration equal to its critical micelle concentration. The emulsion was prepared by operating the magnetic piston at $10 \mathrm{~Hz}$ for 2 min. In Fig. 15, the obtained correlation curves are displayed. The increase in the decay time with increasing waiting time indicates the slowing of the internal dynamics of the emulsion.

The decay in correlation as well as in transmission in backscattering can be described with compressed exponentials; their shape evolves toward more stretched shapes as the emulsion ages. Mean relaxation times in transmission and backscattering geometries were determined from fits of $G(\tau)-1$ using a Kohlrausch-Williams-Watts decay [panel (c)]. For aging times up to $15 \mathrm{~min}$, times measured in transmission and backscattering grow with the same exponential trend. For larger aging time, the two trend starts to diverge; their ratio-which is directly linked to the value of the photon mean free path-increases. This behavior might be attributed to gravity-driven creaming of the emulsion drops, with subsequent increase in their packing fraction in the measurement volume. ${ }^{73,74}$ Inspection of the emulsion at the wop window of the cell gives drop sizes ranging from a few micrometers up to $80 \mu \mathrm{m}$ (see the inset in Fig. 15).

In order to retrieve quantitative information on the average droplet size and on the internal dynamics of the emulsion, the emulsion must be interpreted with the aid of accurate simulations and calibration measurements. These simulations will have to account for a large value of the photon mean free path, which in emulsions might be comparable to the size of the SCU

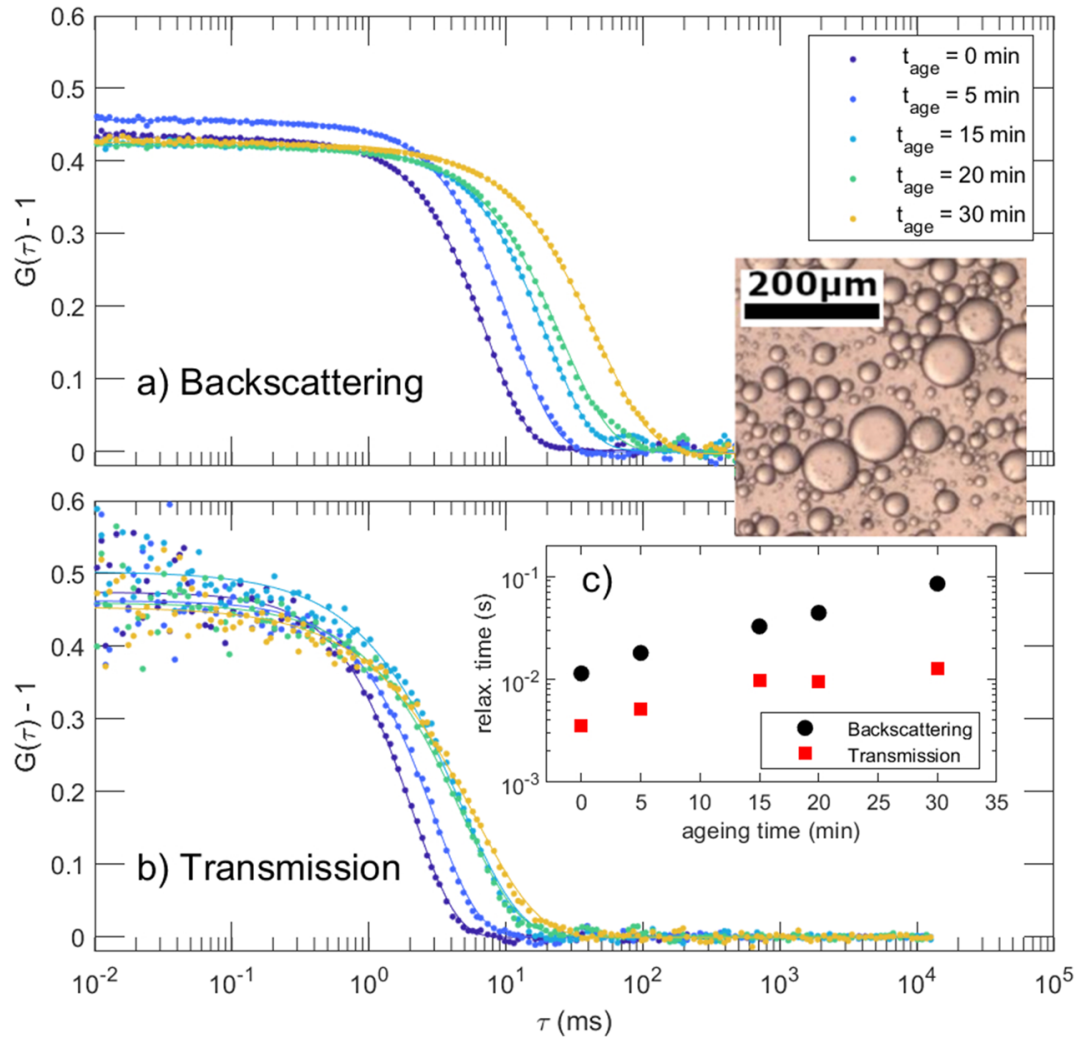

FIG. 15. DWS correlation functions measured on ground on a medium chain triglyceride oil-in-water emulsion stabilized by a food grade surfactant at the critical micelle concentration. Measurements are performed at different ages beyond the end of the emulsification process: (a) backscattering and (b) transmission measurements. The mean relaxation time measured in both geometries increases as a function of aging time [panel (c), determined from fitting with a Kohlrausch-Williams-Watts decay]. The slowing of the dynamics is imputable mainly to the creaming of drops, which increases their packing fraction. The image inset shows an overview image of the emulsion right after emulsification. 
measurement volume as well as for the details of the experimental geometry.

\section{CONCLUSION}

The soft matter dynamics experiment container allows for flexible experiments with a wide range of materials. The realized sample cell units for foams, granular media, and emulsions indicate the variety of research topics that can be covered. Slow dynamics in such soft matter samples, such as coarsening in foams, dynamics in granular media, destabilization of emulsions, and foam rheology, can be studied without gravity over extended time periods, up to months. The design of the container and the modular design of the cells allow us to prepare, process, and manipulate the samples in microgravity. Comprehensive studies on the materials under consideration can be performed with the light scattering diagnostics and the overview camera.

\section{ACKNOWLEDGMENTS}

Many people contributed during the development of the experiment container, the development of the sample cell units, the design of the optical diagnostics, the breadboard tests and the feasibility studies, the coordination among the scientific teams, space administration and industry, the assembly of the container and the sample units, the transport and installation, and the operation of the experiment to and on board the ISS and the analysis of the data. We acknowledge a few representing the many: Hervé Caps (Université de Liège), Laurie Saulnier, and Giles Delon (Université Paris Sud) for performing parabolic flight tests of the SMD hardware with the many foam samples to be studied; Emilie Forel and Maxime Schneider (Université Paris Sud) for performing tests of the cleaning and foaming procedures and implementing the image analysis software; Cecile Gehin Delval (Nestlé) for participating to parabolic flights and supply of chemicals; Denis Weaire, Stefan Hutzler (Trinity College, Dublin), N. Vandewalle (University of Liège), and Alain Cagna (Teclis) for numerous discussions and participations to meetings; and Bernard Zappoli and Christophe Delaroche (CNES) for their continuing support.

The contribution of Jörg Bartnick, Michael Bussmann, Nathan Isert, Georg Maret, Gerold Picker, Liam Redmond, Steffen Reinhold, Johannes Schmitz, and Peidong Yu to the development of the granular media SCU and the diagnostic tests for granular media in laboratory and microgravity settings is gratefully acknowledged.

For the tests on emulsion dynamics experiments in the SMD breadboards and the contribution to the preparation of the incoming related microgravity experiments, we thank Fabrizia Salerni, Angeliki Chondrou, and Robert McMillin for their Ph.D. research work and also acknowledge the contributions of Margaritis Kostoglou, Davide Orsi, Francesca Ravera, Eva Santini, James Ferri, and of all other scientists from academy and industry for participating in the EDDI (Emulsion Dynamics and Droplet Interfaces) MAP project.

Inese Silkina, Luigi Castiglione, Alessandro De Simone, Hans Ranebo, Luciana Montrone, Ana Frutos Pastor, Sebastien Vincent Bonnieu, and Jens Dirk Schiemann contributed to the development and the operation of the experiment on ESA side. We are especially grateful to Olivier Minster (ESA) for his support since the beginning of the project in 1999.
On Airbus side, Stefan Jung, Sergio Krikorian, Gerold Picker, Markus Stauber, and Olaf Schoele-Schulz devoted much of their passion and energy into the realization of the experiment.

Peidong Yu is thanked for proofreading the manuscript.

We acknowledge funding by ESA (via MAP projects "Hydrodynamics of Wet Foams," "Soft Matter Dynamics," and "Emulsion Dynamics and Droplet Interfaces"), CNES, and ASI (project "PASTA-Particle Stabilised Emulsions"). The Federal Ministry for Economic Affairs and Energy (BMWi) and the German Space Agency (DLR) are gratefully acknowledged for their financial support under Grant Nos. 50WM1651, 50WM1652, and 50WM1946 and for securing the realization of the CompGran SCUs. The National Aeronautics and Space Administration (NASA) is also gratefully acknowledged for Grant Nos. NAG3-1419, NAG3-1894, NAG3-2481, NNC04GB61G, NNX07AP20G, NNX14AM99G, 80NSSC19K0599, and 80NSSC21K0898.

\section{AUTHOR DECLARATIONS}

\section{Conflict of Interest}

The authors have no conflicts of interest to disclose.

\section{Author Contributions}

P.B. wrote the frame of the article and the granular cell section and edited the sections provided by the other collaborations, with important contributions by M.B., R.H., D.L., and R.S. All authors edited and validated the manuscript.

S.C.-A., D.D., R.H., D.L., E.R., A.S., and R.S., contributed to the development of the whole experiment container including the light scattering diagnostics and the flexible sample handling approach, which was a prerequisite to the development and usage of the SCUs for all material classes.

S.C.-A., D.D., R.H., D.L., M.P., E.R., A.S., and R.S. contributed to the section on the foam SCU.

P.B., S.U.E., M.A.E.-S., M. Schröter, M. Sperl, and A.B.Z.-B. contributed to the section on the granular media SCU.

L.L., L.C., and T.K. contributed to the section on the emulsion SCU.

R.H. contributed to the section on the foam rheology SCU.

M.B. coordinated the science teams during preparation and execution of the microgravity experiments.

\section{DATA AVAILABILITY}

The data that support the findings of this study are available from the corresponding author upon reasonable request.

\section{REFERENCES}

${ }^{1}$ R. Clift, J. R. Grace, and M. E. Weber, Bubbles, Drops, and Particles (Courier Corporation, 2005).

${ }^{2}$ C. C. Maaß, N. Isert, G. Maret, and C. M. Aegerter, "Experimental investigation of the freely cooling granular gas,” Phys. Rev. Lett. 100, 248001 (2008).

${ }^{3}$ N. Isert, G. Maret, and C. M. Aegerter, "Coarsening dynamics of threedimensional levitated foams: From wet to dry," Eur. Phys. J. E 36, 116 (2013).

${ }^{4}$ T. Steinpilz, G. Musiolik, M. Kruss, F. Jungmann, T. Demirci, M. Aderholz, J. E. Kollmer, J. Teiser, T. Bila, E. Guay et al., “ARISE: A granular matter experiment on the international space station,” Rev. Sci. Instrum. 90, 104503 (2019). 
${ }^{5}$ S. Aumaître, R. P. Behringer, A. Cazaubiel, E. Clément, J. Crassous, D. J. Durian, E. Falcon, S. Fauve, D. Fischer, A. Garcimartín, Y. Garrabos, M. Hou, X. Jia, C. Lecoutre, S. Luding, D. Maza, M. Noirhomme, E. Opsomer, F. Palencia, T. Pöschel, J. Schockmel, M. Sperl, R. Stannarius, N. Vandewalle, and P. Yu, "An instrument for studying granular media in low-gravity environment," Rev. Sci. Instrum. 89, 075103 (2018).

${ }^{6} \mathrm{P}$. Born, J. Schmitz, and M. Sperl, "Dense fluidized granular media in microgravity," npj Microgravity 3, 27 (2017).

${ }^{7}$ S. Mazzoni, M. A. C. Potenza, M. D. Alaimo, S. J. Veen, M. Dielissen, E. Leussink, J.-L. Dewandel, O. Minster, E. Kufner, G. Wegdam, and P. Schall, "SODI-COLLOID: A combination of static and dynamic light scattering on board the International Space Station,” Rev. Sci. Instrum. 84, 043704 (2013).

${ }^{8}$ Y.-P. Chen, P. Evesque, and M.-Y. Hou, "Breakdown of energy equipartition in vibro-fluidized granular media in micro-gravity," Chin. Phys. Lett. 29, 074501 (2012).

${ }^{9}$ A. Saint-Jalmes, S. Marze, M. Safouane, D. Langevin, S. J. Cox, and D. Weaire, "Experiments and simulations of liquid imbibition in aqueous foams under microgravity," Microgravity Sci. Technol. 18, 108-111 (2006).

${ }^{10}$ A. Saint-Jalmes, S. Marze, M. Safouane, and D. Langevin, "Foam experiments in parabolic flights: Development of an ISS facility and capillary drainage experiments," Microgravity Sci. Technol. 18, 22-30 (2006).

${ }^{11}$ S. Manley, L. Cipelletti, V. Trappe, A. E. Bailey, R. J. Christianson, U. Gasser, V. Prasad, P. N. Segre, M. P. Doherty, S. Sankaran, A. L. Jankovsky, B. Shiley, J. Bowen, J. Eggers, C. Kurta, T. Lorik, and D. A. Weitz, "Limits to gelation in colloidal aggregation," Phys. Rev. Lett. 93, 108302 (2004).

${ }^{12}$ Z. Cheng, P. M. Chaikin, J. Zhu, W. B. Russel, and W. V. Meyer, "Crystallization kinetics of hard spheres in microgravity in the coexistence regime: Interactions between growing crystallites," Phys. Rev. Lett. 88, 015501 (2001).

${ }^{13}$ R. Piazza, "Optical correlation techniques for the investigation of colloidal systems," in Colloidal Foundations of Nanoscience (Elsevier, 2014), pp. 233-266.

${ }^{14} \mathrm{P}$. Zakharov and F. Scheffold, "Advances in dynamic light scattering techniques," in Light Scattering Reviews (Springer, Berlin, Heidelberg, 2009), Vol. 4, pp. 433-467.

${ }^{15} \mathrm{~F}$. Scheffold and R. Cerbino, "New trends in light scattering," Curr. Opin. Colloid Interface Sci. 12, 50-57 (2007).

${ }^{16}$ S. U. Egelhaaf, "Solution scattering," in Soft Condensed Matter Physics in Molecular and Cell Biology (Taylor \& Francis, 2006), p. 38.

${ }^{17}$ R. Höhler, S. Cohen-Addad, and D. J. Durian, "Multiple light scattering as a probe of foams and emulsions," Curr. Opin. Colloid Interface Sci. 19, 242-252 (2014).

${ }^{18}$ G. Maret, "Diffusing-wave spectroscopy," Curr. Opin. Colloid Interface Sci. 2, 251-257 (1997).

${ }^{19}$ D. A. Weitz, J. X. Zhu, D. J. Durian, H. Gang, and D. J. Pine, "Diffusing-wave spectroscopy: The technique and some applications," Phys. Scr. T49B, 610-621 (1993).

${ }^{20}$ G. Trinchero, M. Cardano, E. Pensavalle, E. Bassano, P. Dell'Aversana, M. Lappa, and M. Tacconi, "The Fluid Science Laboratory on the ISS Columbus module performances and operations," J. Jpn. Soc. Microgravity Appl. 25, 303 (2008).

${ }^{21}$ D. Weitz and D. Pine, "Diffusing-wave spectroscopy," in Dynamic Light Scattering, Monographs on the Physics and Chemistry of Material, edited by W. Brown (Oxford University Press, 1993), pp. 652-720.

${ }^{22}$ D. J. Pine, D. A. Weitz, P. M. Chaikin, and E. Herbolzheimer, "Features of diffusing wave spectroscopy," Phys. Rev. Lett. 60, 1134-1137 (1988).

${ }^{23}$ L. Cipelletti, H. Bissig, V. Trappe, P. Ballesta, and S. Mazoyer, "Time-resolved correlation: A new tool for studying temporally heterogeneous dynamics," J. Phys.: Condens. Matter 15, S257-S262 (2002).

${ }^{24}$ D. J. Durian, D. A. Weitz, and D. J. Pine, "Multiple light-scattering probes of foam structure and dynamics," Science 252, 686-688 (1991).

${ }^{25} \mathrm{R}$. Sütterlin, "The soft matter dynamics experiment for the ISS," in 69th International Astronautical Congress (IAC 2018), Bremen, Germany (Curran Associates, Inc., 2018), ISBN: 978-1-5108-8165-5, Vol. 1, Chap. IAC-18.A2.6.7.

${ }^{26} \mathrm{D}$. L. Weaire and S. Hutzler, The Physics of Foams (Oxford University Press, 2001).

${ }^{27}$ I. Cantat, S. Cohen-Addad, F. Elias, F. Graner, R. Höhler, O. Pitois, F. Rouyer, and A. Saint-Jalmes, Foams: Structure and Dynamics (OUP, Oxford, 2013).
${ }^{28}$ D. Langevin, Emulsions, Microemulsions and Foams (Springer, 2020).

${ }^{29}$ M. U. Vera, A. Saint-Jalmes, and D. J. Durian, "Scattering optics of foam," Appl. Opt. 40, 4210-4214 (2001).

${ }^{30}$ A. A. Cox and D. J. Durian, "Spatial sampling by diffuse photons," Appl. Opt. 40, 4228-4235 (2001).

${ }^{31}$ A. S. Gittings and D. J. Durian, "Statistics of bubble rearrangement dynamics in a coarsening foam," Phys. Rev. E 78, 066313 (2008).

${ }^{32}$ M. Le Merrer, S. Cohen-Addad, and R. Höhler, "Bubble rearrangement duration in foams near the jamming point," Phys. Rev. Lett. 108, 188301 (2012).

${ }^{33} \mathrm{M}$. Le Merrer, S. Cohen-Addad, and R. Höhler, "Duration of bubble rearrangements in a coarsening foam probed by time-resolved diffusing-wave spectroscopy: Impact of interfacial rigidity," Phys. Rev. E 88, 022303 (2013).

${ }^{34}$ Dynamic Light Scattering: The Method and Some Applications, edited by $\mathrm{W}$. Brown (Clarendon Press, Oxford, 1993).

${ }^{35}$ R. Bandyopadhyay, A. S. Gittings, S. S. Suh, P. K. Dixon, and D. J. Durian, "Speckle-visibility spectroscopy: A tool to study time-varying dynamics," Rev. Sci. Instrum. 76, 093110 (2005).

${ }^{36}$ E. R. Nowak, J. B. Knight, M. L. Povinelli, H. M. Jaeger, and S. R. Nagel, "Reversibility and irreversibility in the packing of vibrated granular material," Powder Technol. 94, 79-83 (1997).

${ }^{37}$ J. B. Knight, C. G. Fandrich, C. N. Lau, H. M. Jaeger, and S. R. Nagel, "Density relaxation in a vibrated granular material," Phys. Rev. E 51, 3957-3963 (1995).

${ }^{38} \mathrm{P}$. Philippe and D. Bideau, "Compaction dynamics of a granular medium under vertical tapping,” Europhys. Lett. 60, 677-683 (2002).

${ }^{39} \mathrm{~L}$. Djaoui and J. Crassous, "Probing creep motion in granular materials with light scattering," Granular Matter 7, 185-190 (2005).

${ }^{40}$ S. Slotterback, M. Toiya, L. Goff, J. F. Douglas, and W. Losert, "Correlation between particle motion and Voronoi-cell-shape fluctuations during the compaction of granular matter," Phys. Rev. Lett. 101, 258001 (2008).

${ }^{41}$ J. H. Schmertmann, "The mechanical aging of soils," J. Geotech. Eng. 117, 1288-1330 (1991).

${ }^{42}$ M. Liingaard, A. Augustesen, and P. V. Lade, "Characterization of models for time-dependent behavior of soils," Int. J. Geomech. 4, 157-177 (2004).

${ }^{43}$ D. J. Scheeres, C. M. Hartzell, P. Sánchez, and M. Swift, "Scaling forces to asteroid surfaces: The role of cohesion," Icarus 210, 968-984 (2010).

${ }^{44}$ L. Cipelletti, S. Manley, R. C. Ball, and D. A. Weitz, "Universal aging features in the restructuring of fractal colloidal gels," Phys. Rev. Lett. 84, 2275-2278 (2000).

${ }^{45}$ L.-O. Heim, J. Blum, M. Preuss, and H.-J. Butt, "Adhesion and friction forces between spherical micrometer-sized particles," Phys. Rev. Lett. 83, 3328-3331 (1999).

${ }^{46}$ M. Kiuchi and A. M. Nakamura, "Relationship between regolith particle size and porosity on small bodies," Icarus 239, 291-293 (2014).

${ }^{47}$ J. P. Marshall, R. C. Hurley, D. Arthur, I. Vlahinic, C. Senatore, K. Iagnemma, B. Trease, and J. E. Andrade, "Failures in sand in reduced gravity environments," J. Mech. Phys. Solids 113, 1-12 (2018).

${ }^{48}$ J. E. Colwell, S. Sture, M. Cintala, D. Durda, A. Hendrix, T. Goudie, D. Curtis, D. J. Ashcom, M. Kanter, T. Keohane, A. Lemos, M. Lupton, and M. Route, "Ejecta from impacts at $0.2-2.3 \mathrm{~m} / \mathrm{s}$ in low gravity,” Icarus 195, 908-917 (2008).

${ }^{49}$ N. Murdoch, I. Avila Martinez, C. Sunday, E. Zenou, O. Cherrier, A. Cadu, and Y. Gourinat, "An experimental study of low-velocity impacts into granular material in reduced gravity," Mon. Not. R. Astron. Soc. 468, 1259 (2017).

${ }^{50} \mathrm{G}$. Caballero, J. Lanuza, and E. Clement, "Compaction and mobility in randomly agitated granular assemblies," in Powders and Grains 2005: Proceedings of the 5th International Conference on Micromechanics of Granular Media, Stuttgart, Germany, 18-22 July, 2005 (Taylor \& Francis, 2005), p. 339.

${ }^{51}$ G. Ovarlez, C. Fond, and E. Clément, "Overshoot effect in the Janssen granular column: A crucial test for granular mechanics,” Phys. Rev. E 67, 060302 (2003).

${ }^{52}$ K. Kim, J. K. Moon, J. J. Park, H. K. Kim, and H. K. Pak, "Jamming transition in a highly dense granular system under vertical vibration," Phys. Rev. E 72, 011302 (2005).

${ }^{53} \mathrm{X}$. Jia, T. Brunet, and J. Laurent, "Elastic weakening of a dense granular pack by acoustic fluidization: Slipping, compaction, and aging," Phys. Rev. E 84, 020301 (2011). 
${ }^{54}$ G. Petekidis, P. N. Pusey, A. Moussaid, S. Egelhaaf, and W. C. K. Poon, "Shearinduced yielding and ordering in concentrated particle suspensions," Physica A 306, 334-342 (2002)

${ }^{55}$ P. A. Smith, G. Petekidis, S. U. Egelhaaf, and W. C. K. Poon, "Yielding and crystallization of colloidal gels under oscillatory shear," Phys. Rev. E 76, 041402 (2007).

${ }^{56}$ A. Amon, A. Mikhailovskaya, and J. Crassous, "Spatially resolved measurements of micro-deformations in granular materials using diffusing wave spectroscopy," Rev. Sci. Instrum. 88, 051804 (2017).

${ }^{57} \mathrm{M}$. van Hecke, "Jamming of soft particles: Geometry, mechanics, scaling and isostaticity," J. Phys.: Condens. Matter 22, 033101 (2009).

${ }^{58}$ B. P. Tighe, "Relaxations and rheology near jamming," Phys. Rev. Lett. 107, 158303 (2011).

${ }^{59} \mathrm{R}$. Höhler and S. Cohen-Addad, "Many-body interactions in soft jammed materials," Soft Matter 13, 1371-1383 (2017).

${ }^{60} \mathrm{R}$. Höhler and D. Weaire, "Can liquid foams and emulsions be modeled as packings of soft elastic particles?,” Adv. Colloid Interface Sci. 263, 19-37 (2019).

${ }^{61}$ M. Schreckenberg and D. Wolf, Traffic and Granular Flow'97 (Springer, Singapore, 1998), Vol. 119.

${ }^{62}$ L. Corté, P. M. Chaikin, J. P. Gollub, and D. J. Pine, "Random organization in periodically driven systems," Nat. Phys. 4, 420-424 (2008).

${ }^{63}$ R. Höhler, S. Cohen-Addad, and H. Hoballah, "Periodic nonlinear bubble motion in aqueous foam under oscillating shear strain," Phys. Rev. Lett. 79, 1154-1157 (1997).

${ }^{64} \mathrm{~T}$. Goloub and R. J. Pugh, "The role of the surfactant head group in the emulsification process: Single surfactant systems," J. Colloid Interface Sci. 257, 337-343 (2003).

${ }^{65}$ S. S. Dukhin, N. A. Mishchuk, G. Loglio, L. Liggieri, and R. Miller, "Coalescence coupling with flocculation in dilute emulsions within the primary and/or secondary minimum," Adv. Colloid Interface Sci. 100-102, 47-81 (2003).
${ }^{66}$ D. Georgieva, V. Schmitt, F. Leal-Calderon, and D. Langevin, "On the possible role of surface elasticity in emulsion stability," Langmuir 25, 5565-5573 (2009).

${ }^{67}$ P. Tchoukov, J. Czarnecki, and T. Dabros, "Study of water-in-oil thin liquid films: Implications for the stability of petroleum emulsions," Colloids Surf., A 372, 15-21 (2010).

${ }^{68}$ B. Deminière, A. Colin, F. L. Calderon, and J. Bibette, "Vieillissement par coalescence et durée de vie d'une emulsion concentrée," C. R. Acad. Sci., Ser. IIc: Chim. 1, 163-165 (1998).

${ }^{69}$ V. I. Kovalchuk, A. V. Makievski, J. Krägel, P. Pandolfini, G. Loglio, L. Liggieri, F. Ravera, and R. Miller, "Film tension and dilational film rheology of a single foam bubble," Colloids Surf., A 261, 115-121 (2005).

${ }^{70} \mathrm{P}$. W. Voorhees, "The theory of Ostwald ripening," J. Stat. Phys. 38, 231-252 (1985).

${ }^{71}$ S. Llamas, E. Santini, L. Liggieri, F. Salerni, D. Orsi, L. Cristofolini, and F. Ravera, "Adsorption of sodium dodecyl sulfate at water-dodecane interface in relation to the oil in water emulsion properties," Langmuir 34, 5978-5989 (2018).

${ }^{72}$ X. Hu, R. Miller, and L. Guo, "Experimental study on interfacial characteristics during bubble dissolution," Colloids Surf., A 505, 179-185 (2016).

${ }^{73}$ F. Salerni, D. Orsi, E. Santini, L. Liggieri, F. Ravera, and L. Cristofolini, "Diffusing wave spectroscopy for investigating emulsions: II. Characterization of a paradigmatic oil-in-water emulsion," Colloids Surf., A 580, 123724 (2019).

${ }^{74}$ D. Orsi, F. Salerni, E. Macaluso, E. Santini, F. Ravera, L. Liggieri, and L. Cristofolini, "Diffusing wave spectroscopy for investigating emulsions: I. Instrumental aspects," Colloids Surf., A 580, 123574 (2019).

${ }^{75}$ V. Lorusso, D. Orsi, F. Salerni, L. Liggieri, F. Ravera, R. McMillin, J. Ferri, and L. Cristofolini, "Recent developments in emulsion characterization: Diffusing wave spectroscopy beyond average values," Adv. Colloid Interface Sci. 288, 102341 (2021). 